\title{
APLICAÇÃO DE ISÓTOPOS ESTÁVEIS DE CARBONO NO ESTUDO DAS MUDANÇAS CLIMÁTICAS E AMBIENTAIS
}

Valdir Felipe NOVELLO

Marília de Carvalho CAMPOS

Cristiano Mazur CHIESSI

Gustavo Macedo de PAULA-SANTOS

Luiz Carlos Ruiz PESSENDA

Taciana GOMES

Giselle UTIDA

Francisco William da CRUZ

\begin{abstract}
RESUMO
O carbono está presente em uma ampla variedade de reservatórios naturais como rochas, solos, oceanos e atmosfera e é um dos principais componentes dos organismos vivos. As razões entre os seus dois isótopos mais abundantes ${ }^{12} \mathrm{C} \mathrm{e}{ }^{13} \mathrm{C}$, descritas pela notação $\delta^{13} \mathrm{C}$, são fundamentais para o entendimento da dinâmica e evolução desses reservatórios. $\mathrm{O}$ fracionamento entre ${ }^{12} \mathrm{C} \mathrm{e}{ }^{13} \mathrm{C}$ ocorre principalmente pela fotossíntese, que incorpora preferencialmente ${ }^{12} \mathrm{C}$ nas moléculas orgânicas, que por sua vez determinam os valores de $\delta^{13} \mathrm{C}$ de grande parte da biota, além de outros materiais. Por exemplo, no caso das plantas, sua assinatura isotópica pode ser transferida ao solo e posteriormente a espeleotemas de cavernas ou a sedimentos transportados pelos rios e depositados nos oceanos. Outro exemplo seria o caso da fotossíntese de algas marinhas que afeta a assinatura isotópica da água do mar, que é transferida para as carapaças carbonáticas de organismos marinhos, tal como foraminíferos. Os valores de $\delta^{13} \mathrm{C}$ desses materiais (i.e., moléculas orgânicas, espeleotemas, foraminíferos) estão intimamente relacionados com as condições climáticas e ambientais nas quais os mesmos se formaram. Nessa revisão, apresentamos como se dá a dinâmica do $\delta^{13} \mathrm{C}$ nas plantas, solos, rochas e oceanos e como o estudo desses reservatórios nos revela informações a respeito do clima e do ambiente do presente e do passado, além de propiciar o entendimento de processos biológicos e geológicos. Os exemplos analisados aqui tratam principalmente de estudos de reconstituições paleoambientais e paleoclimáticas desenvolvidas no território brasileiro e no oceano adjacente.
\end{abstract}

Palavras-chave: $\delta^{13} \mathrm{C}$; Carbono; Isótopos; Paleoambiente; Paleoclima.

\section{ABSTRACT}

CARBON ISOTOPES APPLIED TO STUDIES OF CLIMATE AND ENVIRONMENTAL CHANGES. Carbon is present in a large variety of natural reservoirs, such as rocks, soils, oceans and atmosphere, and is the major component of living organisms. The ratio between their two most common isotopes ${ }^{12} \mathrm{C}$ and ${ }^{13} \mathrm{C}$, denoted as $\delta^{13} \mathrm{C}$, is of great importance in the understanding of the dynamics and evolution of these reservoirs. The fractionation between ${ }^{12} \mathrm{C}$ and ${ }^{13} \mathrm{C}$ mainly occurs during photosynthesis, which preferentially incorporates ${ }^{12} \mathrm{C}$ into organic molecules and controls $\delta^{13} \mathrm{C}$ values of large extents of the biosphere and other materials. For example, the isotopic signature of plants is transferred to soils and later to cave speleothems and/or sediments carried 
by rivers and deposited in the oceans. Another example is the photosynthesis performed by marine algae, which affects the isotopic signature of seawater that is also transferred to carbonate shells of marine organisms, such as foraminifera. Thus, $\delta^{13} \mathrm{C}$ values from these materials (i.e., organic molecules, speleothems, foraminifera) are closely related to the climatic and environmental conditions occurring at their site of formation. In this review, we summarize the dynamics of $\delta^{13} \mathrm{C}$ in plants, soils, rocks and oceans to show how these carbon reservoirs can document present and past climatic and environmental conditions, and therefore, provide important information about biologic and geologic processes. The examples shown in this paper are mainly derived from paleoclimatic and paleoenvironment studies performed in the Brazilian territory and the adjacent ocean.

Keywords: $\delta^{13} \mathrm{C}$; Carbon; Isotopes; Paleoenvironment; Paleoclimate.

\section{INTRODUÇÃO}

Isótopos estáveis de carbono são empregados em uma ampla variedade de estudos ambientais e paleoambientais. Por ser um elemento essencial da vida, o carbono é encontrado em quase todos os compostos orgânicos e sua forma inorgânica está presente em abundância no ar atmosférico e nas águas subterrâneas e oceânicas, acumulando-se em uma ampla variedade de reservatórios naturais. A proporção relativa entre os isótopos do carbono nesses reservatórios pode ser utilizada como um indicador da origem do material, enquanto a variação nos seus valores indica mudança nas condições ambientais às quais esses reservatórios (ou porções específicas dos mesmos) estão sujeitos.

Três isótopos de carbono são comuns na natureza (i.e. ${ }^{12} \mathrm{C},{ }^{13} \mathrm{C} \mathrm{e}{ }^{14} \mathrm{C}$ ), sendo que os isótopos ${ }^{12} \mathrm{C}$ e ${ }^{13} \mathrm{C}$ são estáveis e apresentam abundância relativa aproximada de $98,98 \%$ e $1,10 \%$, respectivamente. $\mathrm{O}$ isótopo radioativo ${ }^{14} \mathrm{C}$ é formado na atmosfera pelo choque entre nêutrons de alta energia (raios cósmicos) e ${ }^{14} \mathrm{~N}$ do ar. $\mathrm{O}{ }^{14} \mathrm{C}$ está presente apenas em concentrações traço nos reservatórios naturais de carbono. Sua meia vida física é de $5.730 \pm 30$ anos e é utilizado para a datação de materiais orgânicos e inorgânicos, sendo fundamental nos estudos que investigam materiais fósseis e sub-fósseis como solos, sedimentos lacustres e marinhos, conchas, corais, madeiras, ossos, entre outros. Nessa revisão trataremos apenas dos isótopos estáveis do carbono, e para efeitos de notação e organização, o carbono na matéria orgânica será referenciado no texto como $\mathrm{C}_{\text {org }}$, o carbono do carbonato como $\mathrm{C}_{\text {carb }}$ e o carbono inorgânico dissolvido como $\mathrm{C}_{\mathrm{CID}}$.

A abundância relativa entre os isótopos estáveis do carbono é representada pela notação $\delta$ (expressa em per mil, \%o), que quantifica a razão
${ }^{13} \mathrm{C} /{ }^{12} \mathrm{C}$ em relação a um padrão internacional de referência com valor conhecido, no caso o Vienna Pee Dee Belemnite (VPDB). O VPDB é uma referência virtual calibrada em relação à composição isotópica de um fóssil carbonático marinho do período Cretáceo da espécie Belemnitella americana. Assim, a abundância do isótopo ${ }^{13} \mathrm{C}$ em relação ao ${ }^{12} \mathrm{C}$ de uma amostra é dada por:

$$
\delta^{13} C=\left(\frac{{\frac{{ }^{13} C}{{ }^{12} C}}_{\text {amostra }}-{\frac{13}{{ }^{12} C}}_{\text {padrão }}}{\frac{13}{12} C_{\text {padrão }}}\right) \times 1000(\%) \quad \text { Eq.(1) }
$$

Portanto, valores maiores de $\delta^{13} \mathrm{C}$ indicam maior abundância relativa do isótopo ${ }^{13} \mathrm{C}$ na amostra analisada em relação ao padrão VPDB, e vice-versa.

Em escala local, os valores de $\delta^{13} \mathrm{C}$ contribuem, por exemplo, para o entendimento da ecofisiologia das plantas, ou seja, como as plantas respondem ao stress hídrico ou diferenças na disponibilidade de luz (BUCHMANN et al. 1997). Em escala continental e global, o $\delta^{13} \mathrm{C}$ pode fornecer informações sobre a fonte e processos de troca de $\mathrm{CO}_{2}$ entre a atmosfera e a biosfera (SUITS et al. 2005). Os isótopos estáveis do carbono são usados para acessar a contribuição relativa de plantas que realizam o ciclo fotossintético $\mathrm{C}_{3}$ e aquelas que realizam o ciclo fotossintético $\mathrm{C}_{4}$ (PESSENDA et al. 2010, HÄGGI et al. 2016), bem como para predizer o material fonte de aerossóis e gases emitidos pela queima de biomassa (MARTINELLI et al. 2002, POWEL et al. 2012).

Naturalmente, os valores de $\delta^{13} \mathrm{C}$ das plantas podem ser associados com alguma modificação na composição dos tecidos e outras partes de animais, e sua assinatura isotópica nestes últimos pode ser utilizada para, por exemplo, identificar a dieta ou 
distinguir níveis tróficos para indivíduos ou populações. Tais estudos foram conduzidos em uma variedade de espécies brasileiras, entre elas: pássaros (HOBSON 2011), peixes (WANTZEN et al. 2002), insetos (MAGNUSSON et al. 1999) bovinos (CERLING et al. 2003), humanos (NARDOTO et al. 2011) e outros primatas (CROWLEY 2012). Os isótopos estáveis do carbono em plantas fósseis e amostras de solo são usados em uma ampla variedade de estudos paleoecológicos. $\mathrm{O} \delta^{13} \mathrm{C}_{\text {org }}$ da matéria orgânica encontrada, por exemplo, no solo e em sedimentos marinhos de sítios influenciados pelo aporte continental é também utilizado para reconstituir o ambiente do passado em termos de mudanças nas comunidades de plantas (VICTORIA et al. 1995; PESSENDA et al. 1998; HÄGGI et al. 2016, 2017; BERTASSOLI et al. 2017, 2019; MULITZA et al. 2017; SUN et al. 2017), mudanças na composição relativa de material lenhoso e vegetação herbácea (CERLING et al. 2011), e mudanças na dinâmica do carbono no solo como resultado da conversão da cobertura vegetal (RHOADES et al. 2000). Por sua vez, espeleotemas de cavernas podem preservar a assinatura do $\delta^{13} \mathrm{C}$ do solo acima da caverna na sua composição carbonática, que pode ser modificada por efeitos da temperatura e hidrologia, propiciando possíveis indicadores para reconstituições ambientais e climáticas (NOVELLO et al. $2019,2021)$. Nos oceanos, o $\delta^{13} \mathrm{C}$ do carbono inorgânico dissolvido $\left(\delta^{13} \mathrm{C}_{\mathrm{CID}}\right)$ na água do mar é registrado no $\delta^{13} \mathrm{C}$ das conchas de organismos marinhos, tais como foraminíferos, constituindo um importante indicador de mudanças pretéritas no ciclo do carbono e circulação oceânica (e.g., DUPLESSY et al. 1984; MULITZA et al. 1998; CAMPOS et al. 2017, 2020; SANTOS et al. 2017a, b; VOIGT et al. 2017).

Portanto, a aplicabilidade das análises de $\delta^{13} \mathrm{C}$ é facilitada devido a sua presença em uma grande quantidade de materiais e reservatórios, o que o torna um indicador frequentemente utilizado em estudos ambientais e paleoambientais. Nesse artigo, apresentamos uma revisão do comportamento dos valores de $\delta^{13} \mathrm{C}$ em diversos reservatórios naturais, onde são explorados os principais condicionantes ambientais e climáticos para o seu fracionamento, com exemplos de estudos realizados no território brasileiro e oceano adjacente. No item 2 é apresentada a dinâmica do $\delta^{13} \mathrm{C}$ em plantas via fotossíntese, o principal meio de retenção de carbono que é transferido posteriormente para outros reservatórios. $\mathrm{O}$ item 3 trata como os valores de $\delta^{13} \mathrm{C}$ são incorporados ao solo e podem ser utilizados para a reconstituição de paleoambientes. No item $4, \mathrm{o} \delta^{13} \mathrm{C}$ é abordado do ponto de vista de suas aplicações em estudos relacionados a agricultura e erosão. No item 5, a dinâmica do $\delta^{13} \mathrm{C}$ é abordada no ambiente de cavernas, onde os espeleotemas conseguem registrar de forma precisa a variação do $\delta^{13} \mathrm{C}$ ao longo do tempo. No item 6, é explicada a dinâmica do $\delta^{13} \mathrm{C}$ no ambiente oceânico e suas implicações para reconstituições paleoclimáticas/paleoambientais a partir dos valores de $\delta^{13} \mathrm{C}$ medidos em foraminíferos. E por último, no item 7, é apresentado como o $\delta^{13} \mathrm{C}$ em rochas é utilizado em estudos sobre a evolução do planeta, revelando ambientes e períodos e explosão e extinção da vida.

\section{DINÂMICA DO $\delta^{13} \mathrm{C}$ EM PLANTAS}

Durante o processo de fotossíntese ocorre a discriminação isotópica nas plantas com relação ao ${ }^{13} \mathrm{CO}_{2}$ (SMITH \& EPSTEIN 1971). Esta discriminação isotópica deve-se às propriedades bioquímicas das enzimas que fixam o carbono atmosférico e o converte em matéria orgânica, e ao processo de difusão que controla a entrada de $\mathrm{CO}_{2}$ nas folhas. Dessa forma, a extensão dessa discriminação é variável pela existência de três tipos diferentes de ciclos fotossintéticos $\left(\mathrm{C}_{3}, \mathrm{C}_{4}\right.$ e CAM $)$ entre as plantas terrestres (BOUTTON 1996).

As plantas com ciclo $\mathrm{C}_{3}$ (Ciclo de Calvin), que compreendem árvores e algumas espécies de gramíneas (Poaceae), reduzem o $\mathrm{CO}_{2}$ em fosfoglicerato por meio da enzima Ribulose-1,5-bifosfato carboxilase/oxigenase (Rubisco), e apresentam valores do $\delta^{13} \mathrm{C}$ entre -32 e -22\%o, com valor médio de $-27 \%$ (Figura 1). Aproximadamente $85 \%$ de todas as espécies de plantas possuem o padrão fotossintético $\mathrm{C}_{3}$ e são dominantes na maioria dos ecossistemas, ocorrendo desde as regiões boreais até os trópicos (BOUTTON 1996, PESSENDA et al. 2005). As plantas $C_{4}$ utilizam a via metabólica Hatch-Slack em conjunto com o Ciclo de Calvin, utilizando a enzima PEP-carboxilase na redução e na fixação do carbono em moléculas orgânicas. Os valores de $\delta^{13} \mathrm{C}$ de plantas do tipo $\mathrm{C}_{4}$ variam entre -9 e $-17 \%$, com média de $-13 \%$ (Figura 1), e são encontrados em sua maioria em gramíneas. As plantas $\mathrm{C}_{4}$ são controladas principalmente pela temperatura, precipitação, disponibilidade de umidade e concentrações de $\mathrm{CO}_{2}$, que mudam a eficiência do processo de fotossíntese (BOUTTON 1996). Embora o metabolismo $C_{3}$ seja predominante em árvores dicotiledôneas e o $\mathrm{C}_{4}$ em gramíneas, há exceções, como, por exemplo, em ambientes 
úmidos (manguezais, turfeiras, etc.), nos quais as ervas apresentam predominantemente o ciclo fotossintético $\mathrm{C}_{3}$ (PESSENDA et al. 2009, 2012).

O padrão de cada tipo de fotossíntese é significantemente influenciado pelas condições climáticas e a distribuição das plantas delimitada pelos padrões ambientais (EHLERINGER et al. 1997). Como os intervalos dos valores isotópicos entre as plantas $\mathrm{C}_{3}$ e $\mathrm{C}_{4}$ não se sobrepõem e por possuírem preferências ecológicas distintas, a análise do $\delta^{13} \mathrm{C}$ de compostos orgânicos produzidos por plantas encontrados em solos e sedimentos continentais é fundamental no estudo das modificações da vegetação ao longo do tempo, tais como as verificadas por MARTINELLI et al. (1996), VIDOTTO et al. (2007), SAIA et al. (2008), PESSENDA et al. (2004, 2009, 2010, 2012), FRANCISQUINI et al. (2014) e BERTASSOLI et al. $(2017,2019)$ em diferentes regiões do Brasil. No caso de sedimentos marinhos coletados em sítios que recebem aporte continental, o $\delta^{13} \mathrm{C}$ de compostos orgânicos produzidos pela vegetação continental pode ser usado tanto no estudo de modificações da vegetação ao longo do tempo como para identificar mudanças das áreas fontes dos sedimentos, indicando, portanto, alterações nos padrões hidroclimáticos pretéritos sobre o continente (HÄGGI et al. 2016, 2017; MULITZA et al. 2017; SUN et al. 2017).

As plantas CAM (Metabolismo do Ácido das Crassuláceas) utilizam tanto a via $\mathrm{C}_{3}$ como a via $\mathrm{C}_{4}$. Essas plantas são capazes de fixar o $\mathrm{CO}_{2}$ durante a noite usando parte da via $\mathrm{C}_{4}$ (PEP-carboxilase), para durante o dia refixarem o $\mathrm{CO}_{2}$ por meio da via $\mathrm{C}_{3}$ (Rubisco). São plantas adaptadas a condições de aridez, como as cactáceas, e possuem valores de $\delta^{13} \mathrm{C}$ intermediários entre os grupos $\mathrm{C}_{3}$ e $\mathrm{C}_{4}$, variando de $-28 \%$ a $-10 \%$ (Figura 1; BOUTTON 1996). Assim, a presença de plantas CAM dificulta os estudos paleoambientais baseados nos valores de $\delta^{13} \mathrm{C}$.

As vias fotossintéticas $\mathrm{C}_{4}$ e CAM são evoluções da fotossíntese $\mathrm{C}_{3}$. A fotossíntese $\mathrm{C}_{4}$ evoluiu em resposta a baixa concentração de $\mathrm{CO}_{2}$ atmosférico, que ocorreu antes do final do período Cretáceo. Baixa concentração de $\mathrm{CO}_{2}$ atmosférico resulta em significativo aumento na fotorrespiração de plantas $\mathrm{C}_{3}$, reduzindo sua produtividade; enquanto que plantas intermediárias (entre $\mathrm{C}_{3}$ e $\mathrm{C}_{4}$ ) e $\mathrm{C}_{4}$, apresentam baixas taxas de fotorrespiração. Por outro lado, plantas CAM parecem ter se desenvolvido em resposta seletiva do aumento da eficiência do uso de água e/ou pelo aumento de ganho de carbono (EHLERINGER \& MONSON 1993). Os três tipos de plantas são atualmente bem distribuídos pelo globo, com uma tendência de adaptação ecológica de plantas $\mathrm{C}_{4}$ dentro de climas mais quentes e de monção, enquanto que as plantas CAM têm melhor adaptação dentro de habitats com disponibilidade hídrica mais limitada.

Em uma escala global, vários estudos mostram que a representatividade das plantas $\mathrm{C}_{4}$ no ambiente é principalmente relacionada à temperatura média da estação de crescimento. Na escala mais regional, a precipitação de verão tem um papel predominante quando a temperatura é mantida constante. De uma forma geral, plantas $\mathrm{C}_{4}$ tendem a ser predominantes em áreas de baixa precipitação ou umidade do solo (EHLERINGER \& MONSON 1993).

Para uma ampla escala geográfica, a distribuição das plantas CAM é claramente influenciada pelo clima árido. Esse tipo de planta apresenta dois grupos principais: suculentas de regiões áridas e semi-áridas, e epífitas de regiões tropicais e subtropicais.

Em uma escala de tempo longa, a eficiência do uso de água por plantas $\mathrm{C}_{3}$ é sensível a diminuição global do $\mathrm{CO}_{2}$ atmosférico, que é maior hoje do que foi durante os períodos glaciais, o que implica que plantas $\mathrm{C}_{3}$ apresentem desvantagens competitivas em períodos glaciais de baixa concentração de $\mathrm{CO}_{2}$ na atmosfera (POLLEY et al. 1992, 1993). Já em um ambiente geneticamente modificado de $\mathrm{CO}_{2}$, com elevados teores na atmosfera, as plantas $\mathrm{C}_{4}$ podem perder sua vantagem competitiva sobre as plantas $\mathrm{C}_{3}$. Plantas $\mathrm{C}_{4}$ também apresentam vantagens competitivas com stress hídrico, enquanto frio e sombra favorece as plantas $C_{3}$ (EHLERINGER 1978).

Árvores dicotiledôneas são predominantemente de metabolismo $\mathrm{C}_{3}$, enquanto o metabolismo $\mathrm{C}_{4}$ é predominante em gramíneas, entretanto, exceções ocorre em ambos os casos. Estudos que levantaram a assinatura isotópica de plantas da Amazônia mostraram que todas as plantas $\mathrm{C}_{4}$ eram gramíneas e todas as plantas $\mathrm{C}_{3}$ eram arbustos ou vinhas, e valores de $\delta^{13} \mathrm{C}$ de plantas dicotiledôneas $(\sim-30 \%)$ eram significantemente mais negativas que as gramíneas mais comuns $(\sim-13 \%)$ (MAGNUSSON et al. 1999).

\section{$3 \delta^{13} \mathrm{C}$ EM SOLOS}

\subsection{Dinâmica de $\delta^{13} \mathrm{C}$ em solos}

A análise de $\delta^{13} \mathrm{C}$ da matéria orgânica do solo, juntamente com a datação pelo método do ${ }^{14} \mathrm{C}$, auxiliam na reconstrução do ambiente vegetacional do 
passado, aspecto fundamental no estudo de paleoclimas. A matéria orgânica das camadas superficiais dos solos possui um valor de $\delta^{13} \mathrm{C}$ característico da vegetação de cobertura. Alguns resultados mostram que o valor do $\delta^{13} \mathrm{C}$ em solos sob plantas $\mathrm{C}_{3}$ está entre $-20 \%$ e $-32 \%$. Em contraste, se a paisagem for aberta, com possibilidades de desenvolvimento de gramínea tropical (ciclo fotossintético $\mathrm{C}_{4}$ ), o $\delta^{13} \mathrm{C}$ estará entre -9\%o e -17\%o (GUILLET et al. 1988). Essa caracterização é especialmente interessante no estudo de savanas, onde ecologistas tem caracterizado mudanças na abundância de árvores ao longo do tempo, uma vez que as gramíneas presentes nas savanas são predominantemente $\mathrm{C}_{4} \mathrm{e}$ a maioria das outras plantas são $C_{3}$. De forma bem geral, a vegetação de savana consiste aproximadamente de uma camada contínua de vegetação rasteira, predominantemente gramíneas, com uma variedade de densidade de árvores e arbustos (KNOOP \& WALKER 1985, GILSON et al. 2004). Assim, valores de $\delta^{13} \mathrm{C}$ podem ser um indicador de tipos de plantas e densidade da vegetação, analisado no âmbito espacial através de transecções entre diferentes tipos de vegetação, e ao longo do tempo quando em perfis de profundidade no solo, caracterizando assim, por exemplo, transições (espaciais e temporais) entre ambientes de campo, savana e floresta (Figura 1).

Os valores de $\delta^{13} \mathrm{C}$ da matéria orgânica do solo próximo a superfície são determinados pela entrada da matéria orgânica proveniente da vegetação atual (BOUTTON et al. 1998). O $\delta^{13} \mathrm{C}$ da matéria orgânica do solo é levemente enriquecido em ${ }^{13} \mathrm{C}$ em relação a biomassa das plantas dominantes $(<=$ $2 \%$ ) (WEDIN et al. 1995), mas tal enriquecimento não é suficiente para mascarar a diferença de $14 \%$ entre a liteira de plantas $\mathrm{C}_{3}$ e $\mathrm{C}_{4}$. Uma vez que a matéria orgânica é preservada no solo, o $\delta^{13} \mathrm{C}$ de camadas mais profundas do solo reflete a composição isotópica da matéria orgânica e vegetação do passado, consequentemente, o $\delta^{13} \mathrm{C}$ de perfis de solo pode ser utilizado como um indicador das mudanças na abundância relativa entre plantas $\mathrm{C}_{3} \mathrm{e}_{4}$ ao longo do tempo (e. g. BOUTTON 1996, BOUTTON et al.1998). Vale destacar que os valores de $\delta^{13} \mathrm{C}$ da matéria orgânica também sofrem alteração em relação a profundidade do solo, ficando mais negativos (empobrecidos em ${ }^{13} \mathrm{C}$ ) em profundidades maiores devido a diferenciação do ${ }^{13} \mathrm{C}$ pela decomposição da matéria orgânica (BECKER-HEIDMANN \& SCHARPENSEEL 1992, NATELHOFFER \& FRY 1988).

A interpretação do $\delta^{13} \mathrm{C}$ do solo pode ser complexa devido a diferente quantidade de compostos produzidos pelas plantas, que é dependente, por exemplo, da sua produtividade e fenologia. Assim, a relação entre o $\delta^{13} \mathrm{C}$ do solo e a assembleia de vegetação não é direta, isso porque a proporção de plantas $\mathrm{C}_{3}$ e $\mathrm{C}_{4}$ da biomassa pode não ser a mesma proporção do carbono fornecido ao solo por cada tipo de planta. Com isso, a origem da matéria orgânica do solo pode ser de difícil caracterização em função da heterogeneidade da vegetação local ou sua densidade relativa. Uma abordagem para a interpretação dos dados de $\delta^{13} \mathrm{C}$ do solo é o método de balanço de massa, que estima a proporção de carbono de origem das plantas $\mathrm{C}_{4} \mathrm{e} \mathrm{C}_{3}$ (e. g. BOUTTON et al. 1998). O princípio por trás da equação do balanço de massa é um modelo de mistura simples, onde as médias dos valores de $\delta^{13} \mathrm{C}$ para plantas $\mathrm{C}_{3}$ e $\mathrm{C}_{4}$ são calculadas por múltiplas amostragens de plantas $\mathrm{C}_{3}$ e $\mathrm{C}_{4}$, e a proporção de carbono originário dos dois tipos de plantas é estimada usando a equação:

$$
\delta^{13} C_{S O M}=\left(\delta^{13} C_{C 4}\right)(x)+\left(\delta^{13} C_{C 3}\right)(1-x)
$$

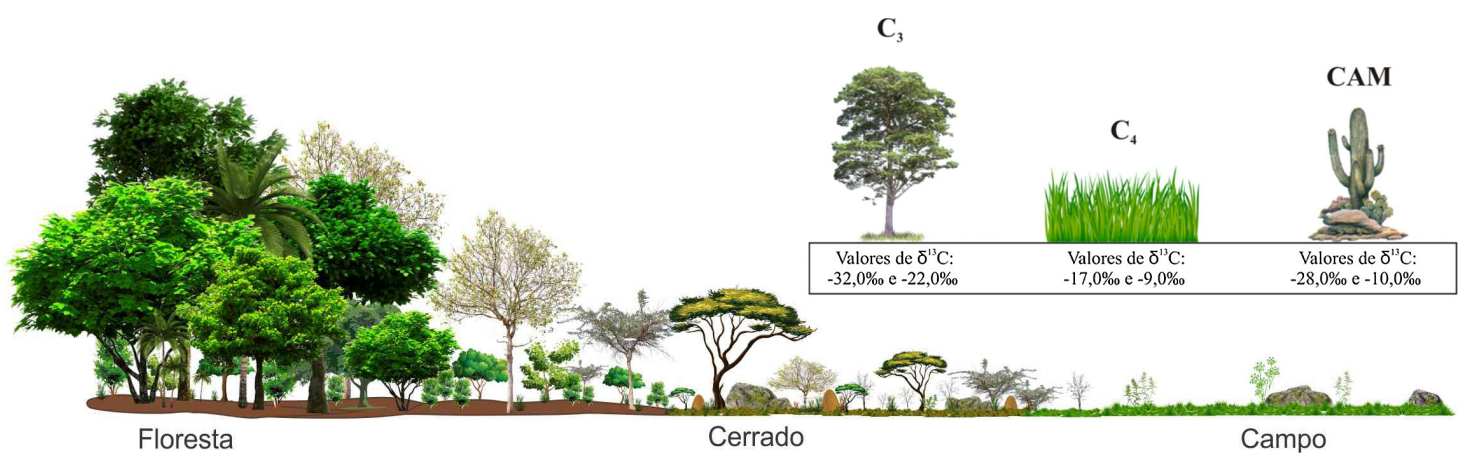

FIGURA 1 - Modelo esquemático indicando o aumento dos valores de $\delta^{13} \mathrm{C}$ no solo na transição entre floresta e campo e intervalos dos valores isotópicos de $\delta^{13} \mathrm{C}$ nos diferentes ciclos fotossintéticos $\left(\mathrm{C}_{3}, \mathrm{C}_{4}\right.$ e CAM). 
Onde o $\delta^{13} \mathrm{C}_{\mathrm{SOM}}$ é o valor medido de $\delta^{13} \mathrm{C}$ na amostra de matéria orgânica do solo, $\delta^{13} \mathrm{C}_{\mathrm{C} 4} \mathrm{e}$ $\delta^{13} \mathrm{C}_{\mathrm{C} 3}$ são as médias dos valores de $\delta^{13} \mathrm{C}$ das plantas $\mathrm{C}_{4}$ e $\mathrm{C}_{3}$, respectivamente. A proporção de carbono que tem como fonte as plantas $\mathrm{C}_{4}$ está representada por $\mathrm{x}$ e, consequentemente, (1-x) é a proporção de carbono originário das plantas $\mathrm{C}_{3}$.

3.2 Reconstituições paleoclimáticas e paleoambientais com o uso de $\delta^{13} \mathrm{C}$ em solos brasileiros

No Brasil, perfis de solo, sedimentos lacustres e sedimentos fluviais foram amplamente estudados com a aplicação de isótopos de carbono, muitas vezes associados a outros marcadores ambientais tais como isótopos de nitrogênio, concentração de carbono orgânico e nitrogênio total na matéria orgânica, fitólitos e pólen (PESSENDA et al. 1996a, 1996b, 1998, 2001a, 2001b, 2004, 2005, 2009, 2010; FREITAS et al. 2001; GOUVEIA et al. 2002; SCHEEL-YBERT et al. 2003; BUSO JR. et al. 2013; FRANCA et al. 2013; CALEGARI et al. 2013, 2017; FRANCISQUINI et al. 2014; ROSSETI et al. 2017). Dentre esses trabalhos, análises de $\delta^{13} \mathrm{C}$ em vários perfis de solo ao longo de várias transecções de 200 a $500 \mathrm{~km}$ realizados em Rondônia e na porção sul do estado do Amazonas indicaram três fases de vegetação na maioria das localidades, que foram caracterizadas por: fase de floresta entre $\sim 17$ e 9 mil anos antes do presente (AP); expansão de savana entre $\sim 9$ e 3 mil anos AP, provavelmente devido a condições climáticas menos úmidas do que a fase anterior; e expansão de floresta a partir de $\sim 3$ mil anos AP (PESSENDA et al. 1998, 2001a; FREITAS et al. 2001), devido ao retorno do clima mais úmido similar ao atual.

Estudos realizados ao longo das regiões Central, Sul e Sudeste do Brasil têm mostrado que a mistura de plantas $\mathrm{C}_{3}$ e $\mathrm{C}_{4}$ prevaleceu na maioria das regiões estudadas durante o período do Holoceno. Na maior parte dos estudos, valores mais enriquecidos (menos negativos) de $\delta^{13} \mathrm{C}$ ocorreram durante o Holoceno inferior e médio, indicando aumento de savana (abertura de floresta), que foi seguido de adensamento da vegetação e aumento de plantas $\mathrm{C}_{3}$ a partir de aproximadamente 4-3 mil anos AP (PESSENDA et al. 2004, 2005; SCHEEL-YBERT et al. 2003; CALEGARI et al. 2013). Os autores têm associado a expansão dos campos (savanização) ocorrida no Holoceno inferior e médio a um clima menos úmido, que foi progressivamente se tornando mais úmido até as condições atuais e propiciou a expansão da cobertura de árvores e ervas $\mathrm{C}_{3}$. Ainda dentro deste cenário, um maior predomínio de plantas $\mathrm{C}_{4}$ (gramíneas) foi reportado nos estados de São Paulo e Paraná desde o Pleistoceno Superior (PESSENDA et al. 1996a,1998, 2004; GOUVEIA et al. 2002). Já em regiões costeiras no Espírito Santo, onde há predomínio da Mata Atlântica, análises de $\delta^{13} \mathrm{C}$ em perfis de solo tem mostrado a predominância de plantas $\mathrm{C}_{3}$ ao longo dos últimos 17 mil anos (BUSO Jr. et al. 2013, CALEGARI et al. 2017), aspectos relacionados a presença de eventuais refúgios florestais associados a campos úmidos (denominados de nativos) dominados por ervas $\mathrm{C}_{3}$.

Solos datados com idades mais antigas que o Holoceno são raros em regiões tropicais, e poucos estudos com o uso da matéria orgânica no solo são existentes para o período do Pleistoceno no Brasil. Entre os estudos existentes, foi documentada a presença de mosaico de gramíneas $\mathrm{C}_{4}$ e $\mathrm{C}_{3}$ (campos) e floresta de altitude na Serra do Mar em São Paulo desde 28 mil anos AP (PESSENDA et al. 2009), e predominância de floresta na porção sul da Amazônia (PESSENDA et al. 2001a) e Nordeste (PESSENDA et al. 2010) para o período entre $\sim 21$ e 12 mil anos AP.

\section{$4 \delta^{13} \mathrm{C}$ E SUAS APLICAÇÕES NA AGRICULTURA}

Pela discussão anterior, nota-se que o $\delta^{13} \mathrm{C}$ também desempenha um papel vital nos estudos agrícolas, uma vez que o carbono é o principal elemento das plantas e da matéria orgânica do solo. O carbono também é protagonista quando se trata de emissão e sequestro de gases de efeito estufa pela agricultura, que irá estocá-lo ou retirá-lo do sistema solo-planta (BAYER et al. 2015, LAL 2004). Assim, a gestão do solo tem papel fundamental na dinâmica dos gases estufa, com aumento de emissão em sistemas agrícolas com preparo convencional do solo e queima de resíduos agrícolas (FANG \& MONCRIEFF 2001, IPCC 2001, LAL 2004), e diminuição de emissão em sistemas agrícolas de baixo carbono, providos de técnicas de manejo e conservação do solo, ou ainda pela formação e manutenção de áreas florestais (LAL 2004, GALDOS et al. 2009, BALDOTTO et al. 2010, MAPA 2012).

A matéria orgânica do solo desempenha papel fundamental no sequestro e estoque de carbono, assim como na manutenção das funções do solo, seja por influenciar a retenção da água, a estrutura, a estabilidade e a biodiversidade do solo, ou por influenciar a fonte de nutrientes para as culturas agrícolas (BRAIDA et al. 2011). As diferentes frações 
granulométricas do solo diferem na dinâmica da matéria orgânica, uma vez que os restos vegetais da cobertura vegetal atual serão refletidos nas frações mais grosseiras do solo, enquanto os vestígios da vegetação mais antiga estarão refletidos nas frações mais finas (CAMPOS 2003, MARTINELLI et al. 2009).

No Brasil, grandes áreas florestais que seguem o ciclo $\mathrm{C}_{3}$ foram transformadas em áreas agropecuárias (MARTINELLI et al. 2010). Atualmente, do total da área utilizada para agropecuária, aproximadamente $72 \%$ são pastagens e $10 \%$ são áreas cultivadas com milho ou cana-de-açúcar, que seguem o ciclo $\mathrm{C}_{4}$, e os demais $18 \%$ são outros usos agrícolas (IBGE 2017). A diferença na assinatura isotópica entre essas culturas pode ser utilizada em diversos estudos, dentre eles os relacionados às diferentes atividades agrícolas estabelecidas em uma área ao longo do tempo (BALESDENT et al. 1987, MARTIN et al. 1990, JASTROW et al. 1996), às diferenças nos estoques de carbono (CAMARGO et al. 1999, ASSAD et al. 2013, OLIVEIRA et al. 2016) e nas emissões de gases do efeito estufa (TRUMBORE et al. 1995, LAPOLA et al. 2013, ZHU et al. 2019) devido às alterações no uso do solo, ou mesmo em estudos relacionados aos processos erosivos (GOMES et al. 2019).

Como exemplo, TARRÉ et al. (2001) demonstrou a variação isotópica do carbono em uma área florestal (i.e. plantas $\mathrm{C}_{3}$ ) convertida para pastagem (i.e. plantas $\mathrm{C}_{4}$ ). Nesse estudo, a matéria orgânica do solo sob floresta, com composição isotópica de $-26 \%$, teve sua composição isotópica enriquecida nos primeiros $30 \mathrm{~cm}$ de profundidade após receber carbono enriquecido em ${ }^{13} \mathrm{C}$ de pastagem por longo período de tempo. Em outros dois estudos realizados em formações predominantemente florestais (i.e. plantas $\mathrm{C}_{3}$ ), VITORELLO et al. (1989) no estado de São Paulo e DESJARDINS et al. (1996) no estado do Amazonas, foi observado que os valores mais negativos de $\delta^{13} \mathrm{C}$ estavam nas frações mais grossas do solo, refletindo a composição isotópica da cobertura vegetal, enquanto as frações mais finas tiveram os valores mais elevados, refletindo a decomposição dessa mesma matéria orgânica. Contrariamente, nas áreas florestais que sofreram alteração no uso do solo para culturas do tipo $\mathrm{C}_{4}$, VITORELLO et al. (1989) e DESJARDINS et al. (1996) notaram que as frações mais grosseiras do solo apresentaram valores mais elevados de $\delta^{13} \mathrm{C}$, refletindo a vegetação atual, enquanto as frações mais finas apresentaram valores relativamente mais negativos de $\delta^{13} \mathrm{C}$, refletindo a vegetação mais antiga.

Através do modelo de mistura isotópica apresentado na Eq. 2 é possível inferir a contribuição de cada vegetação na amostra final (após conversão do uso do solo). Esse mesmo modelo também pode ser utilizado em estudos erosivos, especialmente em microbacias hidrográficas com cultivo de vegetação do tipo $\mathrm{C}_{4}$ e providas de vegetação nativa $\left(\mathrm{C}_{3}\right)$ nas áreas de preservação permanente (APP). Por esse modelo de mistura isotópica, GOMES et al. (2019) identificaram a origem do sedimento depositado e em suspensão nos pequenos riachos que drenam essas áreas, estimando o volume do material erodido por cada uma das áreas a montante do curso d'água, ou seja, qual foi a porcentagem de material proveniente da APP e da área de cana-de-açúcar que chegaram até o curso d'água, causando sedimentação e poluição da água.

Estes são apenas alguns dos inúmeros usos possíveis dos isótopos do carbono em estudos agrícolas e ambientais, que são amplamente utilizados em conjunto com outros isótopos (particularmente o ${ }^{15} \mathrm{~N}$ ) para caracterizar a conversão do uso do solo e outros efeitos antropogênicos.

\section{$5 \delta^{13} \mathrm{C}$ EM REGISTROS DE CAVERNAS}

\subsection{Dinâmica do $\delta^{13} \mathrm{C}$ preservado em estalagmites de cavernas}

Valores de $\delta^{13} \mathrm{C}$ em carbonatos, que é constituinte das estalagmites de cavernas, são obtidos juntamente com dados de $\delta^{18} \mathrm{O}$ a partir de análises em espectrômetros de massa do tipo ICP-MS. Em registros paleoclimáticos de espeleotemas de cavernas, o $\delta^{18} \mathrm{O}$ é preterido nos estudos devido a relação que os isótopos de oxigênio apresentam com processos atmosféricos, especialmente o efeito quantidade (do inglês amount effect), em que valores mais negativos de $\delta^{18} \mathrm{O}$ são associados a maiores quantidade de chuva e vice-versa. Já o $\delta^{13} \mathrm{C}$ é utilizado apenas como um indicador ambiental de importância secundária devido as várias forçantes que influenciam seus valores. Entretanto, recentemente, a associação dos valores de $\delta^{13} \mathrm{C}$ em estalagmites com outros indicadores ambientais se mostrou útil para o estudo de processos erosivos que ocorrem no solo acima das cavernas, reconstituições de paleovegetação, temperatura e hidrologia local (WORTHAM et al. 2017; WARD et al. 2019; NOVELLO et al. 2019, 2021). 
A fonte inicial de carbono para os espeleotemas é o $\mathrm{CO}_{2}$ presente no solo devido a decomposição da matéria orgânica e respiração de raízes. $\mathrm{O}$ $\mathrm{CO}_{2}$ em contato com água forma o ácido carbônico responsável por dissolver a rocha carbonática da caverna, seguindo a equação:

$$
\mathrm{CaCO}_{3}+\mathrm{H}_{2} \mathrm{O}+\mathrm{CO}_{2} \leftrightarrow \mathrm{Ca}^{2+}+2 \mathrm{HCO}_{3}^{-}
$$

A incorporação do carbono na solução que percola as fraturas da caverna pode ocorrer em sistemas abertos ou fechados (HENDY 1971, MCDERMOTT 2004, FOHLMEISTER et al. 2011). Inicialmente, a solução percolante está em equilíbrio com o $\mathrm{CO}_{2}$ do solo, que pode ser considerado um reservatório infinito de carbono para a solução, assim, o bicarbonato $\left(\mathrm{HCO}^{-3}\right)$ da solução adquire a assinatura do $\delta^{13} \mathrm{C}$ do solo. $\mathrm{O}$ permanente contato da solução percolante com o reservatório de carbono do solo caracteriza um sistema aberto, o que mantém o equilíbrio isotópico entre ambos. Nessas condições, o carbono da rocha é pouco incorporado à solução e sua influência nos valores de $\delta^{13} \mathrm{C}$ do carbono inorgânico dissolvido (CID) pode ser negligenciada. Já em um sistema fechado, a solução percolante perde contato com o reservatório de $\mathrm{CO}_{2}$ do solo, e o $\mathrm{CO}_{2}$ da solução é progressivamente consumido durante o processo de dissolução da rocha da caverna. Assim, a incorporação de carbono da rocha passa a ser significativa nos valores de $\delta^{13} \mathrm{C}_{\mathrm{CID}}$. Na maioria dos casos, a interação entre a solução percolante e a rocha da caverna ocorre em um sistema misto.

Os valores de $\delta^{13} \mathrm{C}$ do carbono pedogênico está relacionado aos valores isotópicos da vegetação predominante (CERLING 1984, QUADE et al. 1989) e são transferidos parcialmente para as estalagmites precipitadas dentro das cavernas a partir da degaseificação (escape de $\mathrm{CO}_{2}$ ) da solução que percolou até a zona vadosa. Esse processo segue a reação inversa da dissolução da rocha apresentada pela equação 3. Dessa forma, estalagmites provenientes de cavernas de um ambiente onde a vegetação externa é dominada por plantas $\mathrm{C}_{3}$ terá valores de $\delta^{13} \mathrm{C}$ entre -14 e $-6 \%$, enquanto cavernas de ambientes com predomínio de plantas $\mathrm{C}_{4}$ terá estalagmites com valores de $\delta^{13} \mathrm{C}$ entre $-6 \mathrm{e}$ +2\% (DREYBRODT 1988, BAKER et al. 1997, MCDERMOTT 2004).

No epicarste e na zona vadosa da caverna pode ocorrer uma precipitação primária de calcita (PPC) devido à perda de $\mathrm{CO}_{2}$ da solução em contato com o ar. Esse processo deixa a solução remanescente mais rica em ${ }^{13} \mathrm{C}$ que será incorpora- do às estalagmites (MICKLER et al. 2019). A PPC é mais frequente durante períodos secos devido à maior exposição da solução percolante a bolsas de ar ao longo do epicarste, resultando na maior perda de $\mathrm{CO}_{2}$ por parte da solução. Dessa forma, eventos de PPC estão diretamente associados às condições hidrológicas. Em regiões de intensa atividade convectiva, como é o caso da maior parte das regiões tropicais do Brasil, o efeito quantidade é o processo que domina o fracionamento do $\delta^{18} \mathrm{O}$ da água da chuva, onde altos índices pluviométricos estão associados à diminuição dos valores de $\delta^{18} \mathrm{O}$ (VUILLE et al. 2012). Esse aumento de chuva pode também causar a diminuição nos valores de $\delta^{13} \mathrm{C}$ das estalagmites, devido a diminuição da taxa de PPC e/ou pelo aumento da umidade no solo que elevam os índices de respiração da vegetação. Assim, esses fatores, quando atendidos, levam a uma correlação positiva entre os valores de $\delta^{18} \mathrm{O}$ e $\delta^{13} \mathrm{C}$ das estalagmites (CRUZ et al. 2006, MICKLER et al. 2006).

A preservação do sinal de $\delta^{13} \mathrm{C}$ da solução de gotejamento nas estalagmites ocorre se a precipitação das mesmas ocorrer em equilíbrio isotópico. Entretanto, o desequilíbrio isotópico fraciona cinematicamente o $\delta^{13} \mathrm{C}$ no momento da formação da estalagmite, esse desequilíbrio é favorecido com a ventilação da caverna, que por sua vez é dependente da temperatura. Esse efeito é mais comum em cavernas localizadas em latitudes mais altas sujeitas a maior sazonalidade das estações. Já em cavernas de regiões tropicais, o fracionamento propiciado por temperaturas entre $15^{\circ} \mathrm{e} 30^{\circ} \mathrm{C}$ são inferiores a $0,5 \%$ no $\delta^{13} \mathrm{C}$ (POLAG et al. 2010).

5.2 Reconstituições paleocimáticas e ambientais com o uso de $\delta^{13} \mathrm{C}$ em registros de cavernas brasileiras

$\mathrm{O}$ registro mais longo de $\delta^{13} \mathrm{C}$ em estalagmites brasileiras documentou variações isotópicas ao longo de mais de 100 mil anos a partir da estalagmite BT2, proveniente da caverna Botuverá, estado de Santa Catarina (CRUZ et al. 2006). Esse estudo mostrou que o $\delta^{13} \mathrm{C}$ da estalagmite BT2 esteve relacionado a produtividade de $\mathrm{CO}_{2}$ do solo, que é modulado em escala orbital pelas mudanças de temperatura e umidade do solo decorrentes da mudança entre regimes climáticos (CRUZ et al. 2006). Foi sugerido que a predominância do regime de monção durante a estação de verão resulta em condições mais quentes e úmidas elevando a produtividade de $\mathrm{CO}_{2}$ no solo acima da caverna, o que leva a diminuição os valores de $\delta^{13} \mathrm{C}$ nas estalagmites. Por 
outro lado, os valores mais positivos são relacionados ao impacto negativo do esfriamento atmosférico na produção biogênica de $\mathrm{CO}_{2}$, resultado da maior atividade de frentes frias e secas características do regime de inverno.

Um estudo realizado com o uso de $\delta^{13} \mathrm{C}$ em estalagmites e sedimento aprisionado dentro da caverna Jaraguá, localizada no município de Bonito no Mato Grosso do Sul, mostrou que o período do último glacial foi dominado por um ambiente de vegetação esparsa com predomínio de plantas $\mathrm{C}_{4}$, pobre em matéria orgânica e com pouco solo acima da caverna devido a erosão mais intensa (NOVELLO et al. 2019). Esse cenário contrastou com o ambiente do Holoceno, quando se desenvolveu uma vegetação mais densa com predomínio de plantas $\mathrm{C}_{3}$ que fixou o solo acima da caverna, elevando os teores de matéria orgânica nesse solo. Essa transformação, que se deu durante o último deglacial, foi evidenciada por ambos os registros de $\delta^{13} \mathrm{C}$ (i.e. estalagmites e sedimentos da caverna), que mostraram uma diferença em torno de $10 \%$ nos valores isotópicos entre os períodos glacial (mais positivo) e Holoceno (mais negativo). Essa interpretação foi reforçada por outros indicadores ambientais medidos nas estalagmites e no sedimento, tais como análises de ${ }^{87} \mathrm{Sr} /{ }^{86} \mathrm{Sr}$ nas estalagmites e a química e quantidades totais de carbono orgânico e inorgânico medidos no perfil sedimentar escavado na caverna (Figura 2, NOVELLO et al. 2019).
Outros estudos mostraram que o $\delta^{13} \mathrm{C}$ de estalagmites de cavernas da América do Sul reflete principalmente as condições hidrológicas locais (JAQUETO et al. 2016, WORTHAM et al. 2017, AZEVEDO et al. 2019, NOVELLO et al. 2021). Essa relação foi evidenciada principalmente a partir de um estudo envolvendo uma compilação com séries de $\delta^{13} \mathrm{C}$ de 25 estalagmites precipitadas ao longo dos últimos 2 mil anos (NOVELLO et al. 2021), onde foi verificada correlação significativa dos valores de $\delta^{13} \mathrm{C}$ com o $\delta^{18} \mathrm{O}$ e a quantidade de chuva local. Nesse estudo, também foi mostrado que a temperatura é o segundo fator mais influente na variação dos valores $\delta^{13} \mathrm{C}$ das estalagmites, e que plantas do tipo $\mathrm{C}_{3}$ foram mais abundantes nas regiões cársticas estudadas ao longo dos últimos milênios.

A relação entre o $\delta^{13} \mathrm{C}$ e a hidrologia local já tinha sido apontada em estudos prévios de estalagmites brasileiras (JAQUETO et al. 2016, WORTHAM et al. 2017, AZEVEDO et al. 2019). Para a estalagmite ALHO6 da caverna Pau d'Alho, localizada no estado do Mato Grosso (JAQUETO et al. 2016), os autores mostraram uma relação entre a concentração de minerais magnéticos com os valores de $\delta^{13} \mathrm{C}$ devido a dinâmica do solo e vegetação acima da caverna. Períodos mais secos, e valores de $\delta^{13} \mathrm{C}$ mais positivos, são associados por JAQUETO et al. (2016) a um solo menos estável acima da caverna, o que resulta em alta erosão e aumento do fluxo de minerais para dentro do sistema
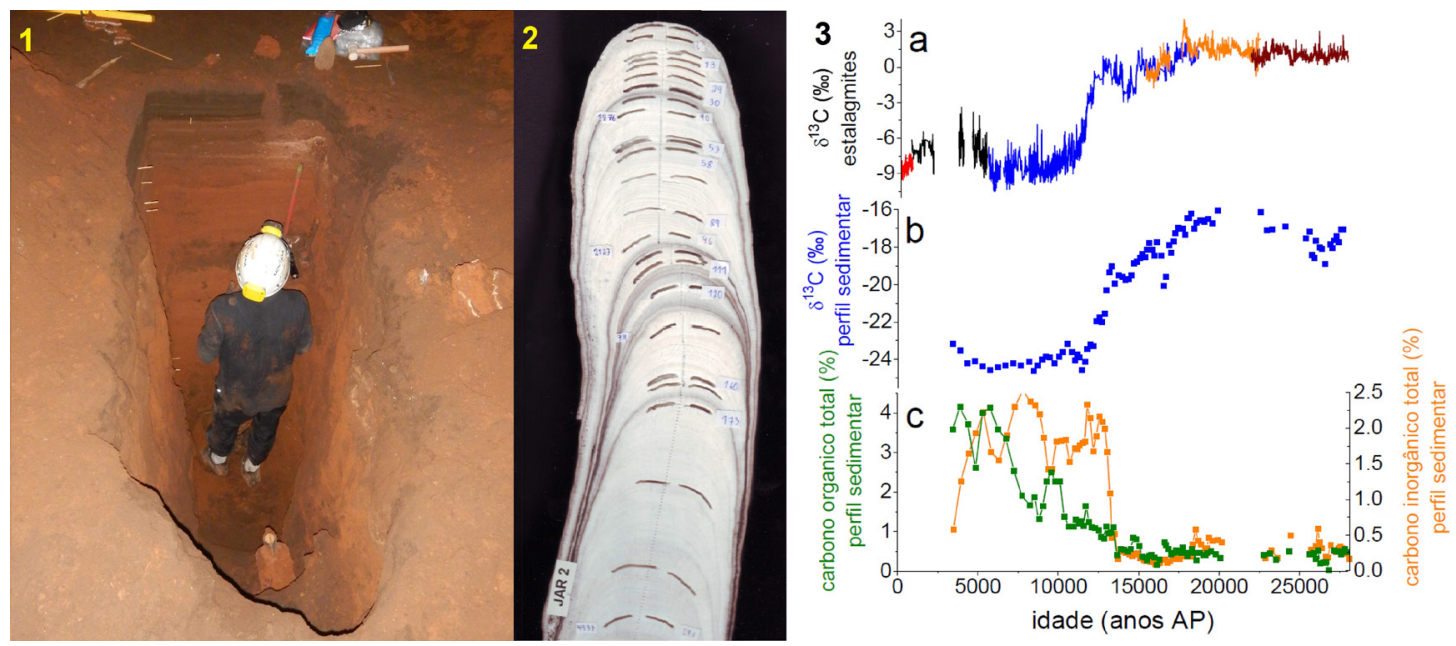

FIGURA 2 - 1- Perfil sedimentar escavado na caverna Jaraguá (Bonito-MS); 2- Face de uma estalagmite da caverna Jaraguá; 3- Registros paleoclimáticos/paloambientais obtidos a partir do sedimento e estalagmites da caverna Jaraguá: (a) perfil de $\delta^{13} \mathrm{C}$ das estalagmites (cada cor é representativa de uma estalagmite), (b) perfil de $\delta^{13} \mathrm{C}$ da matéria orgânica do perfil sedimentar, (3) séries de carbono orgânico total e carbono inorgânico total do perfil sedimentar (modificado de NOVELLO et al. 2019). 
cárstico, enquanto períodos úmidos, com valores baixos de $\delta^{13} \mathrm{C}$, são associados a solos cobertos por vegetação mais densa que retém minerais pedogênicos, reduzindo o influxo desses para a caverna.

\section{$6 \delta^{13} \mathrm{C}$ NOS OCEANOS}

6.1 Dinâmica do $\delta^{13} \mathrm{C}_{\mathrm{CID}}$ nos oceanos e fatores controladores do $\delta^{13} \mathrm{C}$ dos foraminíferos

Os oceanos desempenham um papel fundamental no sistema climático terrestre, a partir, por exemplo, da absorção e redistribuição de calor e $\mathrm{CO}_{2}$. Eles armazenam aproximadamente $88 \%$ do carbono de ciclagem rápida presente na superfície terrestre. Adicionalmente, cerca de $1 / 3$ do $\mathrm{CO}_{2}$ emitido pelas atividades antrópicas é absorvido pelos oceanos (ROMANOU et al. 2017), amenizando marcantemente os impactos das mudanças climáticas (IPCC 2019, RAVEN et al. 2005). O $\delta^{13} \mathrm{C}_{\mathrm{CID}}$ da água do mar é um importante indicador do ciclo do carbono e tem papel de destaque no entendimento de mudanças climáticas.

A distribuição do $\delta^{13} \mathrm{C}_{\mathrm{CID}}$ nos oceanos é controlada (i) pelo balanço fotossíntese/respiração, (ii) por mudanças na área de formação e circulação das massas de águas e (iii) por trocas gasosas oceano-atmosfera (KROOPNICK 1985, SARMIENTO et al. 1988, MULITZA et al. 1999).

Enquanto a fotossíntese remove preferencialmente ${ }^{12} \mathrm{C}$ das águas superficiais, deixando-as com maior $\delta^{13} \mathrm{C}_{\mathrm{CID}}$, a respiração (ou remineralização) da matéria orgânica retorna ${ }^{12} \mathrm{C}$ para as águas profundas, deixando-as com menor $\delta^{13} \mathrm{C}_{\mathrm{CID}}$ (SARMIENTO et al. 1988). Assim, uma fotossíntese mais (menos) intensa leva à maior (menor) exportação de carbono da superfície para o fundo e, portanto, está relacionada com uma "bomba biológica" mais forte (fraca) e maior (menor) diferença entre o $\delta^{13} \mathrm{C}_{\mathrm{CID}}$ das águas superficiais e profundas dos oceanos. O balanço fotossíntese/ respiração também influencia diretamente a distribuição de nutrientes nos oceanos, que apresenta relação inversa com o $\delta^{13} \mathrm{C}_{\mathrm{CID}}$ na coluna de água, i.e. maior $\delta^{13} \mathrm{C}_{\mathrm{CID}}$ e menor concentração de nutrientes nas águas superficiais e menor $\delta^{13} \mathrm{C}_{\mathrm{CID}}$ e maior concentração de nutrientes nas águas profundas (KROOPNICK 1985, HILLAIRE-MARCEL \& RAVELO 2007).

A circulação das massas de água pode sobrepor-se ao balanço fotossíntese/respiração na distribuição do $\delta^{13} \mathrm{C}_{\mathrm{CID}}$. No Atlântico, a massa de água chamada Água Profunda do Atlântico Norte
(APAN) é formada nas altas latitudes do Atlântico Norte por águas superficiais oligotróficas e, portanto, apresenta baixo conteúdo de nutrientes e ${ }^{12} \mathrm{C}$, resultando em valores de $\delta^{13} \mathrm{C}_{\mathrm{CID}}$ relativamente altos $(\sim 1 \%$ ) (KROOPNICK 1985). Esse sinal se espalha junto com essa massa de água em direção ao Atlântico Sul. A APAN é envelopada pela Água Intermediária Antártica (AIA) e pela Água Antártica de Fundo (AAF), ambas possuem conteúdo de nutrientes e ${ }^{12} \mathrm{C}$ alto e, portanto, valores relativamente baixos de $\delta^{13} \mathrm{C}_{\mathrm{CID}}(\sim 0,5 \%$ e $0,4 \%$, respectivamente). Elas são originadas no Oceano Austral e se deslocam para norte em profundidades intermediárias (i.e. AIA) e profundas (i.e. AAF). Conforme o tempo de residência das massas de água que não estão em contato com a atmosfera aumenta, os nutrientes e o carbono remineralizado se tornam mais concentrados, produzindo assinaturas de $\delta^{13} \mathrm{C}_{\mathrm{CID}}$ mais negativas (KROOPNICK 1985, MULITZA et al. 1999).

A interação oceano-atmosfera também afeta o $\delta^{13} \mathrm{C}_{\mathrm{CID}}$. Durante as trocas entre esses dois reservatórios ocorre o fracionamento isotópico do carbono, pois o ${ }^{13} \mathrm{C}$ é mais solúvel que $\mathrm{o}{ }^{12} \mathrm{C}$. A intensidade do fracionamento depende principalmente da temperatura da superfície do mar, mas também é afetada pela taxa de troca de $\mathrm{CO}_{2}$ e pelo tempo de residência das águas na interface oceano-atmosfera (LYNCH-STIEGLITZ et al. 1995, MULITZA et al. 1999). Um exemplo da interação oceano-atmosfera está relacionado com as emissões antrópicas de $\mathrm{CO}_{2}$ advindas da queima de combustíveis fósseis, que são ricos em ${ }^{12} \mathrm{C}$. Essas emissões promovem uma diminuição no $\delta^{13} \mathrm{C}$ do $\mathrm{CO}_{2}$ atmosférico, que por sua vez, ao interagir com o oceano, transmite esse sinal para as águas superficiais (especialmente em locais de formação de massas de água) no curto prazo, e para o oceano profundo no longo prazo (EIDE et al. 2017). Esse é o chamado "Efeito Suess" (KEELING 1979).

Além dos efeitos de escala global, o $\delta^{13} \mathrm{C}_{\mathrm{CID}}$ também pode ser influenciado por processos locais, tais como ressurgência, descarga de rios e emanações de metano (MACKENSEN et al. 1993, THEODOR et al. 2016, PORTILHO-RAMOS et al. 2018).

Assim como outros grupos de organismos marinhos, os foraminíferos (protozoários marinhos unicelulares que constituem um dos principais componentes dos carbonatos marinhos) usam o $\delta^{13} \mathrm{C}_{\mathrm{CID}}$ da água do mar para construir seu esqueleto de carbonato de cálcio (comumente chamado de testa). Desta forma, a composição isotópica do 
carbono das testas dos foraminíferos depende fundamentalmente da composição isotópica do CID da água do mar na qual as testas foram formadas (ROHLING \& COOKE 1999). No entanto, tal relação só é verdadeira se o desvio com relação ao equilíbrio é conhecido (WEFER \& BERGER 1991, ROHLING \& COOKE 1999). Para tanto, são utilizados estudos envolvendo foraminíferos coletados em armadilhas de sedimentos em suspensão (e.g., VENANCIO et al. 2017), foraminíferos depositados no período recente (e.g., RAVELO \& FAIRBANKS 1995, CHIESSI et al. 2007, STEPH et al. 2009), foraminíferos cultivados vivos (e.g., SPERO 1992, MACKENSEN et al. 1993) e realizados experimentos com precipitados de calcita inorgânica (e.g., ROMANEK et al. 1992).

Tais estudos mostraram que o $\delta^{13} \mathrm{C}$ dos foraminíferos é um excelente indicador do $\delta^{13} \mathrm{C}_{\mathrm{CID}}$, no entanto, é preciso conhecer os hábitos de vida das espécies (e.g. profundidade de calcificação na coluna de água, ciclo de vida), as propriedades da massa de água na qual elas calcificaram (e.g. águas corrosivas, zonas de ressurgência, descarga de rios) e ter cautela com efeitos vitais que promovem o fracionamento isotópico espécie-específico durante a biomineralização. Os efeitos vitais são diretamente relacionados com o metabolismo das espécies, a presença de simbiontes e o fracionamento cinético (SPERO \& LEA 1993, RAVELO \& FAIRBANKS 1995, SPERO et al. 1997, MULITZA et al. 1999).

6.2 Uso do $\delta^{13} \mathrm{C}$ de foraminíferos para reconstituições paleoambientais

O uso de foraminíferos em estudos paleoclimáticos/paleoceanográficos teve início na década de 1940, mas apenas três décadas depois a composição dos isótopos estáveis do carbono da calcita de foraminíferos fósseis tornou-se uma ferramenta amplamente utilizada para reconstituir a história ambiental da Terra (SHACKLETON 1977, HILLAIRE-MARCEL \& RAVELO 2007, MACKENSEN \& SCHMIEDL 2019). Além do alto potencial em preservar de maneira mais ou menos direta o $\delta^{13} \mathrm{C}_{\mathrm{CID}}$ do momento e local de formação das suas testas, foraminíferos são excelentes indicadores paleoambientais, pois apresentam marcante abundância nos sedimentos marinhos, excelente preservação e possuem habitats conhecidos (GUPTA 1999).

$\mathrm{O} \delta^{13} \mathrm{C}$ de foraminíferos planctônicos e bentônicos têm sido aplicados de diversas formas para compreender as mudanças paleoambientais no ciclo do carbono. Por exemplo, a composição isotópica de uma ou mais espécies de foraminíferos que calcificam em profundidades distintas e conhecidas é usada em diversas escalas de tempo para reconstituir a concentração de nutrientes ao longo da coluna de água e investigar a distribuição e proporção relativa de massas de água superficiais, intermediárias e profundas (e.g., DUPLESSY et al. 1984, MULITZA et al. 1998, SANTOS et al. 2017a, b; VOIGT et al. 2017, CAMPOS et al. 2017, 2020).

O estudo de MULITZA et al. (1998) foi um dos pioneiros a usar o $\delta^{13} \mathrm{C}$ de foraminíferos para tratar da paleoprodutividade do Atlântico Sul com base em três espécies de foraminíferos, duas planctônicas e uma bentônica. O foco principal foi entender como a concentração de nutrientes na coluna de água variou nos últimos $\sim 400 \mathrm{mil}$ anos. Os autores mostraram que o gradiente de $\delta^{13} \mathrm{C}$ entre a camada de mistura e a termoclina diminuiu nos períodos frios (i.e. glaciais e subestágios frios dos interglaciais), o que eles atribuíram à uma redução na concentração de nutrientes da termoclina (aumento dos valores de $\delta^{13} \mathrm{C}$ ), em virtude da redistribuição de nutrientes para as águas profundas (redução dos valores de $\delta^{13} \mathrm{C}$ ). Tal sugestão deu suporte à teoria que invoca o oceano profundo como o principal reservatório de acúmulo do carbono que deixou a atmosfera durante períodos de resfriamento da Terra (BROECKER \& PENG 1993).

$\mathrm{O} \delta^{13} \mathrm{C}$ de foraminíferos planctônicos também foi usado em estudos recentes que focaram no papel desempenhado pela Célula de Revolvimento Meridional do Atlântico (CRMA) em períodos-chave, tais como transições glacial-interglacial (e.g. SANTOS et al. 2017a) e eventos abruptos de escala milenar (i.e. Heinrich Stadials) (e.g. CAMPOS et al. 2017). Mudanças na intensidade da CRMA são comumente associadas a mudanças marcantes no sistema climático global, e.g. variações na concentração de $\mathrm{CO}_{2}$ atmosférico (Figura 3d, g), na distribuição de calor e salinidade superficiais, na intensidade da ressurgência no Oceano Austral, na "bomba biológica" oceânica e no vazamento das Agulhas. CAMPOS et al. (2017) e SANTOS et al. (2017a) usaram o $\delta^{13} \mathrm{C}$ de espécies planctônicas para rastrear mudanças na Água Central do Atlântico Sul (ACAS) relacionadas com variações na intensidade da CRMA (Figura 3a, b). Os autores demonstraram que, durante momentos de CRMA enfraquecida (e.g. Heinrich Stadials e última transição glacia-interglacial, respectivamente), um dos fatores controladores das excursões negativas em $\delta^{13} \mathrm{C}$ de espécies planctônicas que calcificam sua testa na ACAS esteve associado com uma ressurgência mais inten- 
sa no Oceano Austral. Uma vez que as águas formadas nesse oceano são precursoras da ACAS, uma ressurgência mais intensa (caracterizada por valores relativamente mais baixos de $\delta^{13} \mathrm{C}_{\mathrm{DIC}}$ ) teria promovido a disseminação desse sinal mais negativo para latitudes médias do Atlântico Sul.

Os foraminíferos bentônicos também são muito utilizados para tratar da CRMA em diversas escalas de tempo. Por exemplo, ao analisar o $\delta^{13} \mathrm{C}$ de espécies bentônicas que calcificam sua testa na APAN (em conjunto com outros paleoindicadores), foi identificado que períodos marcados por excursões negativas de $\delta^{13} \mathrm{C}$ em escala milenar e orbital em profundidades intermediárias e médias do Atlântico estão relacionados com o aumento do tempo de residência da APAN (redução da convecção na sua área de formação) e consequente acúmulo de carbono remineralizado nessa massa de água (Figura 3e, f) (LUND et al. 2015, OPPO et al. 2015, HOWE et al. 2016, SANTOS et al. 2017b; VOIGT et al. 2017, CAMPOS et al. 2020). Ainda nessas escalas de tempo, o $\delta^{13} \mathrm{C}$ de foraminíferos bentônicos também ajudou a correlacionar momentos de redução da intensidade da CRMA com aumentos na precipitação sobre o norte do Nordeste do Brasil em escala milenar (Figura 3c) (MULITZA et al. 2017) e variações no estoque/liberação de calor em latitudes médias do Atlântico Sul em escala orbital (Figura 3f) (SANTOS et al. 2017b).
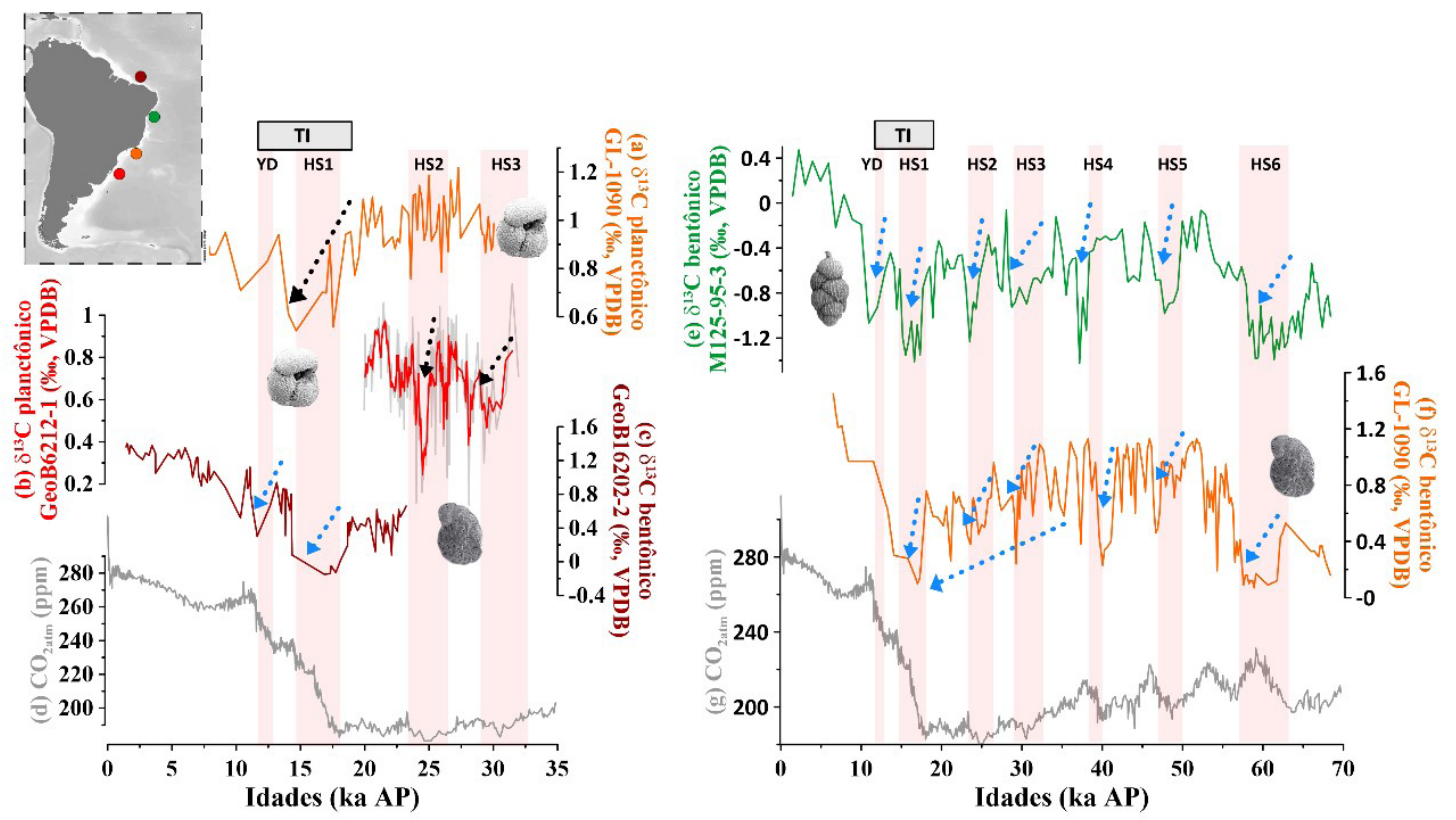

$\therefore \begin{aligned} & 0^{*} \text { ressugência } \\ & \text { mais intensa } \quad \therefore \text { maior tempo de }\end{aligned}$

FIGURA 3 - Registros paleoceanográficos/paleoclimáticos de $\delta^{13} \mathrm{C}$ e $\mathrm{CO}_{2}$ para os últimos ca. 70 mil anos. (a) $\delta^{13} \mathrm{C}$ de foraminífero planctônico do testemunho sedimentar marinho GL-1090 (Atlântico Sul subtropical - ca. $25^{\circ} \mathrm{S}$ ) (SANTOS et al. 2017a); (b) $\delta^{13} \mathrm{C}$ de foraminífero planctônico do testemunho sedimentar marinho GeoB6212-1 (Atlântico Sul subtropical - ca. 32 ${ }^{\circ}$ S) (CAMPOS et al. 2017); (c) $\delta^{13} \mathrm{C}$ de foraminífero bentônico do testemunho sedimentar marinho GeoB16202-2 (Atlântico Sul equatorial - ca. ${ }^{\circ}$ S) (VOIGT et al. 2017); (d) concentração de $\mathrm{CO}_{2}$ atmosférico $\left(\mathrm{CO}_{2 \mathrm{~atm}}\right)$ obtida a partir de uma compilação de testemunhos de gelo (KÖHLER et al. 2017); (e) $\delta^{13} \mathrm{C}$ de foraminífero bentônico do testemunho sedimentar marinho M125-95-3 (Atlântico Sul tropical - ca. $11^{\circ} \mathrm{S}$ ) (CAMPOS et al. 2020); (f) $\delta^{13} \mathrm{C}$ de foraminífero bentônico do testemunho sedimentar marinho GL-1090 (Atlântico Sul subtropical - ca. 25² $)$ (SANTOS et al. 2017b); (g) concentração de $\mathrm{CO}_{2 \mathrm{~atm}}$ obtida a partir de uma compilação de testemunhos de gelo (KÖHLER et al. 2017). O mapa contém a localização (círculos coloridos) dos registros de $\delta^{13} \mathrm{C}$ apresentados na figura (a escala de cor dos círculos está de acordo com as curvas dos respectivos registros de $\delta^{13} \mathrm{C}$ ). As imagens das espécies de foraminíferos utilizadas foram adicionadas próximas de cada respectivo registro de $\delta^{13} \mathrm{C}$. As barras em rosa indicam os intervalos de recorrência dos eventos Heinrich Stadials (HS6-YD). Os retângulos cinza representam o intervalo de recorrência da Terminação I (TI - última transição glacial-interglacial). 


\section{$7 \delta^{13} \mathrm{C}$ NA INVESTIGAÇÃO DA EVOLUÇÃO DA TERRA}

As composições isotópicas do carbono registradas em rochas depositadas ao longo do tempo geológico constituem informações-chave no entendimento de eventos tectono-climáticos ao longo da história da Terra. Rochas sedimentares depositadas em ambientes marinhos são os principais arquivos das variações ocorridas no reservatório global de carbono. A evolução do fracionamento isotópico do carbono entre a matéria orgânica e minerais carbonáticos nestas rochas fornece informações sobre a fração de matéria orgânica soterrada nos sedimentos antes de ser oxidada e, consequentemente, sobre o potencial oxi-redutor da superfície do planeta, bem como a $p \mathrm{CO}_{2}$ atmosférica e a produtividade primária do ambiente marinho (HAYES et al. 1999, KUMP \& ARTHUR 1999).

Em estudos de rochas sedimentares antigas, em que a resolução temporal do estudo abrange intervalos de milhões de anos, assume-se que os diversos processos de feedback negativo dentro do ciclo do carbono do planeta e o equilíbrio termodinâmico entre o $\mathrm{CO}_{2}$ atmosférico e o CID no oceano mantém o reservatório global em estado estacionário. Desta forma o balanço isotópico e de massas podem ser descritos pela equação:

$$
\delta^{13} \mathrm{C}_{\text {entrada }}=\delta^{13} \mathrm{C}_{\text {saida }}
$$

Na qual $\delta^{13} \mathrm{C}_{\text {entrada }}$ é a composição isotópica dos fluxos adentrando a atmosfera e a hidrosfera. Em geral, assume-se o valor de -5\%, que é a média global aferida na Terra moderna (HAYES et al. 1999). $\delta^{13} C_{\text {saida }}$ corresponde à fração de carbono retirado do sistema na forma de minerais carbonáticos ou de matéria orgânica sedimentar. Desta forma, $\delta^{13} \mathrm{C}_{\text {saida }}$ pode ser desmembrado da seguinte forma:

$$
\delta^{13} \mathrm{C}_{\text {entrada }}=\left(1-f_{\text {org }}\right) \times \delta^{13} \mathrm{C}_{\text {carb }}+f_{\text {org }} \times \delta^{13} \mathrm{C}_{\text {org }} \quad \text { Eq. (5) }
$$

Na qual $f_{\text {org }}$ é fração de carbono soterrado como matéria orgânica. Considerando $\delta^{13} \mathrm{C}_{\text {entrada }}$ constante a $-5 \%$, a fração de carbono orgânico soterrado em sedimentos é descrita pela equação:

$$
f_{\text {org }}=\left(\delta^{13} \mathrm{C}_{\text {carb }}+5\right) /\left(\delta^{13} \mathrm{C}_{\text {carb }}-\delta^{13} \mathrm{C}_{\text {org }}\right) \quad \text { Eq. }(6)
$$

Pela equação 6 , nota-se a estreita relação entre $f_{\text {org }}$ e os valores de $\delta^{13} \mathrm{C}_{\text {org }}$ e $\delta^{13} \mathrm{C}_{\text {carb. }}$. A alta bioprodutividade primária tende a aumentar $f_{\text {org }} \mathrm{e}$ o valor de $\delta^{13} \mathrm{C}_{\text {carb }}$, visto que o carbonato se precipita com a composição isotópica do líquido residual. De maneira inversa, se as condições do ambiente marinho forem oxidantes, a matéria orgânica pode ser oxidada liberando novamente ${ }^{12} \mathrm{C}$ para água do mar, diminuindo $f_{\text {org }}$ e o valor de $\delta^{13} \mathrm{C}_{\text {carb }}$. Portanto, os valores de $\delta^{13} \mathrm{C}_{\text {carb }}$ medidos em rochas sedimentares marinhas antigas são basicamente um balanço entre fixação de carbono na matéria orgânica e preservação/oxidação da mesma, o que pode ser usado em interpretações de diversos eventos geológicos de grande magnitude.

Exemplos desta aplicação são os eventos de anoxia do oceano (OAE - Ocean anoxic events) ocorridos no Cretáceo. Induzidos possivelmente por uma alta $p \mathrm{CO}_{2}$ atmosférica e equilíbrio com o oceano, grandes quantidades de carbono foram fixadas na matéria orgânica por microorganismos marinhos, o que rapidamente exauriu o oxigênio da água do mar, levando à anoxia e deposição de espessas sucessões de folhelhos negros e de calcários com $\delta^{13} \mathrm{C}_{\text {carb }}$ bastante positivos (e.g. JENKYNS 1985, DAVEY \& JENKYNS 1999, KASHIYAMA et al. 2008). Estes eventos registrados pelos isótopos de carbono têm grande relevância na história do planeta, visto que marcam um dos grandes eventos de extinção em massa. Registros climáticos de níveis de $p \mathrm{CO}_{2}$ atmosférico ainda mais elevados são registrados em carbonatos biogênicos e abióticos durante as extinções em massa do limite Permiano-Triássico (ver revisão em KORTE \& KOZUR 2010). Inicialmente, os elevados níveis de $\mathrm{CO}_{2}$ na atmosfera desencadearam um grande aumento do intemperismo de crosta continental, que descarregou altas quantidades de ${ }^{12} \mathrm{C}$ oriundo de matéria orgânica continental para o oceano, evento registrado em excursões negativas de $\delta^{13} \mathrm{C}_{\text {carb }}$ da ordem de 4-7\%o. Acima do limite PermianoTriássico, nota-se uma incursão de $\delta^{13} \mathrm{C}_{\text {carb }}$ para valores positivos, que marca a ampla fixação de carbono na matéria orgânica por fotossíntese, gerando anoxia da água do mar e preservação dessa matéria orgânica.

É importante salientar que estas interpretações assumem que o fracionamento do carbono no oceano é quase que exclusivamente realizado pela fotossíntese. Nos casos acima, isto foi atestado pela obtenção de valores de $\delta^{13} \mathrm{C}_{\text {org }}$ nas rochas estudadas, que apresentaram variações acopladas àquelas de $\delta^{13} \mathrm{C}_{\text {carb }}$, resultando em um fracionamento constante entre carbonato e matéria orgânica $\left(\delta^{13} \mathrm{C}=\delta^{13} \mathrm{C}_{\text {carb }}-\delta^{13} \mathrm{C}_{\text {org }}\right)$. Entretanto, existem registros de ampla variação de $\delta^{13} \mathrm{C}$ em rochas antigas, que requerem outras causas para explicar o 
fracionamento além da fotossíntese. Um exemplo são as marcadas excursões negativas de $\delta^{13} C_{\text {carb }}$ em rochas carbonáticas depositadas imediatamente acima de rochas de ambiente glacial durante o Neoproterozoico. Tais depósitos sugerem alternâncias climáticas extremas entre períodos glaciais e de efeito estufa intenso (HOFFMAN \& SCHRAG 2002). Os valores de $\delta^{13} C_{\text {carb }}$ caem para $\sim-5 \%$ nos calcários e dolomitos imediatamente acima dos depósitos glaciais, se recuperando para valores levemente positivos acima na estratigrafia (revisão em HALVERSON et al. 2005). Entretanto, os valores de $\delta^{13} \mathrm{C}_{\text {org }}$ nestas rochas descrevem um comportamento antitético, gerando ampla variação nos valores de $\delta^{13} \mathrm{C}$ (e.g., JIANG et al. 2010, SANSJOFRE et al. 2011, CAETANO-FILHO et al. 2020). Diversas hipóteses foram elaboradas para explicar tal contraste, tal como mudanças no fator de fracionamento causado pela fotossíntese, absorção de $\mathrm{HCO}_{3}{ }^{-}$durante este processo ou fluxo significativo de carbono alóctone para a bacia, mas a real causa permanece em debate.
Independente do mecanismo de fracionamento em si, é notável que assinaturas isotópicas de carbono marcantes são encontradas não só nos períodos anteriormente citados, mas também em outros episódios da história do Planeta, como em glaciações Paleoproterozoicas (BEKKER et al. 2001), no término do Mesoproterozoico (KAH et al. 2012), e no Carbonífero, quando elevada $p \mathrm{CO}_{2}$ na atmosfera foi modelada a partir de valores de $\delta^{13} \mathrm{C}$ (BERNER et al. 2000). Além da interpretação dos processos geológicos ocorridos nestes períodos, o fato de que assinaturas marcantes são encontradas em diversas partes da Terra permite o uso dos isótopos de carbono para correlação de seções geológicas distantes de mesma idade (Figura 4). Os horizontes geológicos com valores distintos de $\delta^{13} \mathrm{C}$ podem ser correlacionados, refinando o posicionamento temporal das seções estudadas e permitindo uma integração global dos dados para entender de maneira ampla as mudanças ocorridas no Sistema Terra em um determinado período. Em alguns casos, até mesmo uma idade relativa pode ser atribuída à seção geológica estudada. Entretanto,

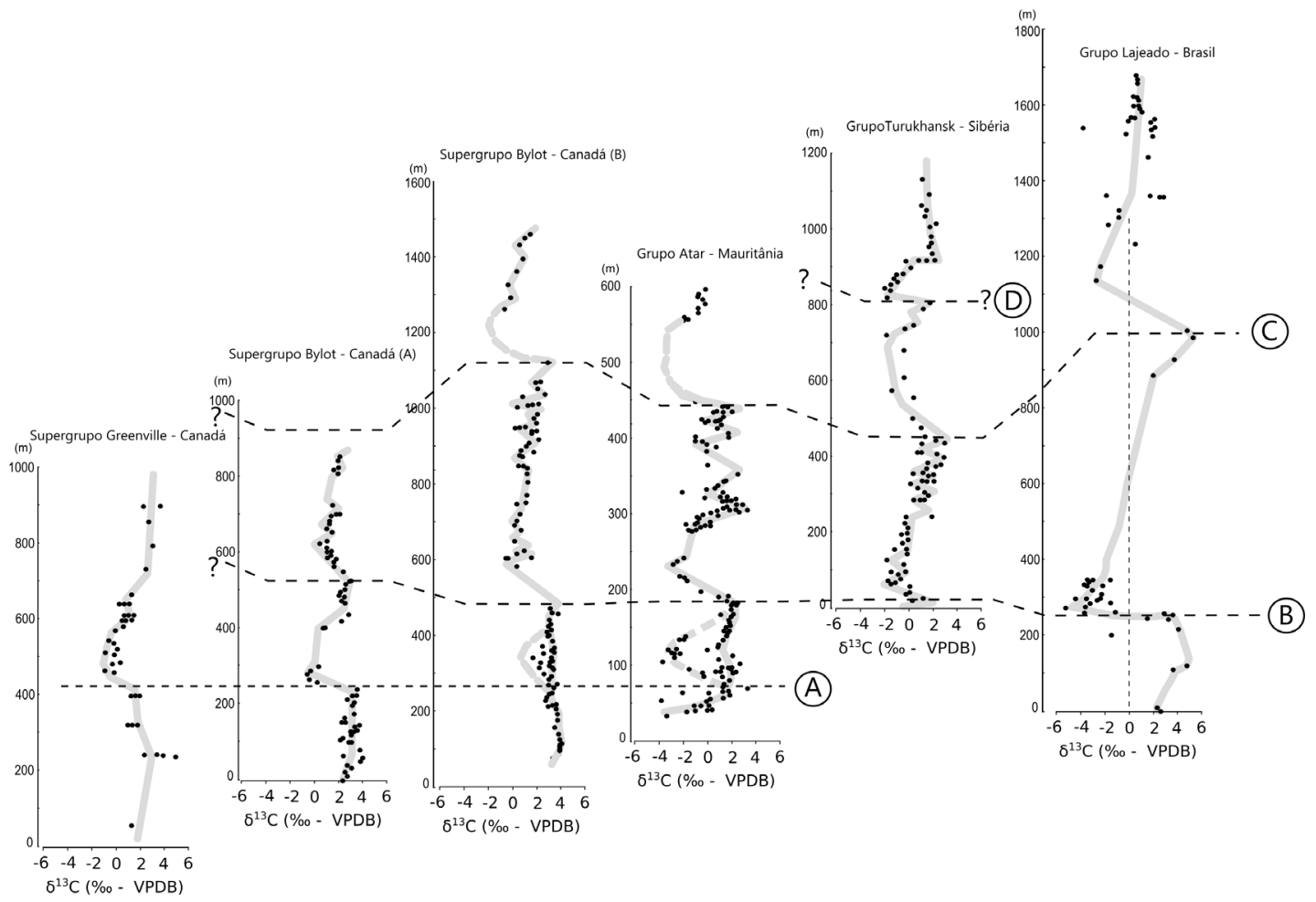

FIGURA 4 - Correlação quimioestratigráfica baseada em valores de $\delta^{13} \mathrm{C}_{\text {carb }}$ obtidos em rochas carbonáticas depositadas entre 1,1-1,2 Ga (modificada de KAH et al. 2012; extraída de PAULA-SANTOS et al. 2021). Os horizontes A, B, C e D foram definidos exclusivamente com base nos dados isotópicos de carbono, independente de quaisquer outras técnicas estratigráficas. Note que assinaturas similares de $\delta^{13} \mathrm{C}$ ocorrem no Canadá, Brasil, Mauritânia e Sibéria. 
sugere-se cautela nesta aplicação, visto que uma mesma assinatura isotópica de carbono se repete diversas vezes no registro geológico. O posicionamento temporal de seções geológicas não deve ser feito apenas baseada em valores de $\delta^{13} \mathrm{C}$, mas sim com base em um conjunto mais amplo de dados que envolve bioestratigrafia, geocronologia e até mesmo quimioestratigrafia isotópica baseada em outros sistemas isotópicos, tendo os isótopos de carbono como uma das ferramentas envolvidas.

Por fim, um ponto de grande relevância no uso de isótopos de carbono em rochas antigas é a possível alteração pós-deposicional dos valores de $\delta^{13} \mathrm{C}$ nelas registrados. Rochas depositadas há centenas de milhões ou até mesmo alguns bilhões de anos atrás passaram por diversos processos geológicos como diagênese, maturação térmica e metamorfismo. Embora o carbono seja constituinte majoritário tanto da matéria orgânica, quanto dos minerais carbonáticos, o que o torna mais resiliente a possíveis modificações, estes processos podem alterar as assinaturas primárias de $\delta^{13} \mathrm{C}_{\text {org }}$ e $\delta^{13} \mathrm{C}_{\text {carb }}$ (KNAUTH \& KENNEDY 2009, DERRY 2010). Isto pode levar a interpretações paleoambientais imprecisas ou até mesmo a erro de correlação de seções. Desta forma, recomenda-se que o uso de isótopos de carbono em rochas antigas seja acompanhado de estudos minuciosos de estratigrafia, sedimentologia e petrografia, além de outros dados geoquímicos que assegurem o caráter primário não-alterados dos valores de $\delta^{13} \mathrm{C}$.

\section{CONCLUSÃO}

$\mathrm{O} \delta^{13} \mathrm{C}$ é utilizado em uma ampla diversidade de estudos ambientais e paleoambientais. Nesse trabalho, apresentamos os principais processos ambientais que controlam o fracionamento e a preservação do sinal isotópico do carbono em reservatórios naturais, com ênfase nas reconstituições paleoambientais e paleoclimáticas do Brasil e do oceano adjacente. Apesar de bastante útil, na maioria dos estudos, os valores de $\delta^{13} \mathrm{C}$ precisam vir acompanhadas de outros indicadores ambientais para permitir sua interpretação robusta, uma vez que ele é sensível a diversas forçantes.

Por ser um indicador relacionado a processos biológicos, as análises de $\delta^{13} \mathrm{C}$ em rochas carbonáticas indicam grandes mudanças planetárias ocorridas ao longo da história do planeta, principalmente aquelas relacionadas com a explosão e retração da vida que influenciaram processos climáticos e geológicos. Nos oceanos, o $\delta^{13} \mathrm{C}$ das testas de fora- miníferos registra o $\delta^{13} \mathrm{C}_{\mathrm{CID}}$ e, assim, é amplamente usado como indicador do balanço fotossíntese/respiração, mudanças na área de formação e circulação das massas de água e interações oceano-atmosfera, contribuindo para o melhor entendimento das mudanças no ciclo do carbono e no clima. No continente, especialmente no Brasil, análises de $\delta^{13} \mathrm{C}$ em solos vem proporcionando reconstituições de vegetação com a base na abundância relativa entre plantas $\mathrm{C}_{3} \mathrm{e} \mathrm{C}_{4}$ e densidade arbórea de savanas, $\mathrm{e}$, mais recentemente, também vem sendo aplicado em espeleotemas para caracterização de processos erosivos, mudanças na vegetação e no ciclo hidrológico.

\section{AGRADECIMENTOS}

Agradecemos o suporte financeiro da Fundação de Amparo à Pesquisa do Estado de São Paulo (FAPESP) a partir de bolsas de pesquisa a V.F.N. (processo 2016/15807-5), G.U. (processo 2020/02737-4) e a M.C.C. (processo 2019/25179-0), além de projeto temático a F.W.C. (processo 2017/50085-3) e projeto jovem pesquisador (fase 2) a C.M.C. (processo 2018/151234). C.M.C. ainda agradece ao apoio financeiro da Coordenação de Aperfeiçoamento de Pessoal de Nível Superior (CAPES) (processos 564/2015 e 88881.313535/2019-01), do Conselho Nacional de Desenvolvimento Científico e Tecnológico (CNPq) (processo 302607/2016-1) e da Alexander von Humboldt Foundation.

\section{REFERÊNCIAS}

ASSAD, E.D.; PINTO, H.S.; MARTINS, S.C.; GROPPO, J.D.; SALGADO P.R.; EVANGELISTA, B.; VASCONCELLOS, E.; SANO, E.E.; PAVÃO, E.; LUNA, R.; CAMARGO, P.B.; MARTINELLI, L.A. 2013. Changes in soil carbon stocks in Brazil due to land use: paired site comparisons and a regional pasture soil survey. Biogeosciences, 10(10): 6141-6160. https://doi.org/10.5194/ bg-10-6141-2013

AZEVEDO, V.; STRÍKIS, N.M.; SANTOS, R.A.; SOUZA, J.G.; AMPUERO, A.; CRUZ, F.W.; OLIVEIRA, P.; STUMPF, C.F.; VUILLE, M.; MENDES, V.R.; CHENG, H.; EDWARDS, R.L. 2019. Medieval Climate Variability in the eastern Amazon-Cerrado regions and its archeological implications. Scientific 
Reports, 9: 20306. https://doi.org/10.1038/ s41598-019-56852-7

BAKER, A.; ITO, E.; SMART, P.L.; MCEWAN, R.F. 1997. Elevated and variable values of $\mathrm{d} 13 \mathrm{C}$ in speleothems in a British cave system. Chemical Geology, 136(3-4): 263-270. https://doi.org/10.1016/S00092541(96)00129-5

BALDOTTO, M.A.; CANELA, M.C.; CANELLAS, L.P.; DOBBSS, L.B.; VELLOSO A.C.X. 2010. Redox index of soil carbon stability. Revista Brasileira de Ciências do Solo, 34(5): 1543-1551. https:// doi.org/10.1590/S0100-06832010000500007

BALESDENT, J.; MARRIOTTI, A.; BERNARD, G. 1987. Natural ${ }^{13} \mathrm{C}$ abundance as a tracer for studies of soil organic matter dynamics. Soil Biology and Biochemistry, 19(1): 25-30. https://doi.org/10.1016/00380717(87)90120-9

BAYER, C.; ZSCHORNACK, T.; PEDROSO, G.M.; DA ROSA, C.M.; CAMARGO, E.S.; BOENI, M.; MARCOLIN, E.; DOS REIS, C.E.S.; DOS SANTOS, D.C. 2015. A sevenyear study on the effects of fall soil tillage on yield-scaled greenhouse gas emission from flood irrigated rice in a humid subtropical climate. Soil and Tillage Research, 145: 118-125. https://doi.org/10.1016/j. still.2014.09.001

BEKKER, A.; KAUFMAN, A.J.; KARHU, J.A.; BEUKES, N.J.; SWART, Q.D.; COETZEE, L.L.; ERIKSSON, K.A. 2001. Chemostratigraphy of the Paleoproterozoic Duitschland Formation, South Africa: Implications for Coupled Climate Change and Carbon Cycling. American Journal of Sciences, 301(3): 261-285. https://doi. org/10.1017/S0033822200063797

BECKER-HEIDMANN, P.; SCHARPENSEEL, H.W. 1992. The use of natural $14 \mathrm{C}$ and $13 \mathrm{C}$ in soils for studies on global climate change. Radiocarbon, 34(3): 535-540. https://doi. org/10.1017/S0033822200063797

BERNER, R.A.; PETSCH, A.T.; BEERLING, D.J.; POPP, B.N.; LANE, R.S.E.A.; LAWS, E.A.; WESTLEY, M.B.; CASSAR, N.; WOODWARD, F.I.; QUICK. W.P. 2000.
Isotope Fractionation and Atmospheric Oxygen: Implications for Phanerozoic O2 Evolution. Science, 287(5458): 1630-1633. https://doi.org/10.1126/science.287.5458.1630

BERTASSOLI JR, D. J.; SAWAKUCHI, A. O.; SAWAKUCHI, H. O.; PUPIM, F. N.; HARTMANN, G.A.; MCGLUE, M.M.; CHIESSI, C.M.; ZABEL, M.; SCHEFU $\beta$, E.; PEREIRO,T.S.; SANTOS, R.A.;FAUSTINO, S.B.; OLIVEIRA, P.E.; BICUDO, D.C. 2017. The fate of carbon in sediments of the Xingu and Tapajós clearwater rivers, eastern Amazon. Frontiers in Marine Science, 4:44. https://doi.org/10.3389/fmars.2017.00044

BERTASSOLI JR, D.; SAWAKUCHI, A.; CHIESSI, C.M.; SCHEFUß, E.; HARTMANN, G.A.; HÄGGI, C.; CRUZ, F.W.; ZABEL, M.; MCGLUE, M.M.; SANTOS, R.A.; PUPIM, F.N. 2019. Spatiotemporal variations of riverine discharge within the Amazon Basin during the late Holocene coincide with extratropical temperature anomalies. Geophysical Research Letters, 46(15): 9013-9022. https:// doi.org/10.1029/2019GL082936

BOUTTON, T. 1996. Stable carbon isotope ratios of soil organic matter and their use as indicators of vegetation and climate change. In: T.W. Boutton \& S.-I. Yamasaki (ed.) Mass spectrometry of soils. New York, Marcel Dekker, p. 47-82.

BOUTTON, T.; ARCHER, S.R.; MIDWOOD, A.J.; ZITZER, S.F; BOL, R. 1998. d13C values of soil organic carbon and their use in documenting vegetation change in a subtropical savanna ecosystem. Geoderma, 82(1-3): 5-41. https://doi.org/10.1016/ S0016-7061(97)00095-5

BRAIDA. J.A.; BAYER, C.; ALBUQUERQUE. J.A.; REICHERT, J.M. 2011. Matéria orgânica e seu efeito na física do solo. Sociedade Brasileira em Ciência do Solo. Tópicos em Ciência do Solo, 7: 221-278.

BROECKER, W.S.; PENG, T.H. 1993. Evaluation of the ${ }^{13} \mathrm{C}$ constraint on the uptake of fossil fuel $\mathrm{CO}_{2}$ by the ocean. Global biogeochemical cycles, 7(3): 619-626. https://doi.org/10.1029/93GB01445 
BUCHMANN, N.; GUEHL, J.-M.; BARIGAH, T.S.;EHLERINGER, J. R. 1997. Interseasonal comparison of $\mathrm{CO}_{2}$ concentrations, isotopic composition and carbon dynamics in an Amazonian rainforest (French Guiana). Oecologia, 110: 120-131. https://doi. org/10.1007/s004420050140

BUSO JR. A.A.; PESSENDA, L.C.R.; DE OLIVEIRA, P.E.; GIANNINI, P.C.F.; COHEN, M.C.L.; VOLKMER-RIBEIRO, C.; OLIVEIRA, S.M.B.; ROSSETTI, D.F.; LORENTE, F.L.; BOROTTI FILHO, M.A.; SCHIAVO, J.A.; BENDASSOLLI, J.A.; FRANÇA, M.C.; GUIMARÃES, J.T.F.; SIQUEIRA, G.S. 2013. Late Pleistocene and Holocene vegetation, climate dynamics, and Amazonian taxa in the Atlantic Forest, Linhares, SE Brazil. Radiocarbon, 55 (23): 1747-1762. https://doi.org/10.1017/ S0033822200048669

CAETANO-FILHO, S.; SANSJOFRE, P.; ADER, M.; PAULA-SANTOS, G.M.; GUACANEME, C.; BABINSKI, M.; BEDOYA-RUEDA, C.; KUCHENBECKER, M.; REIS, H.L.S.; TRINDADE, R.I.F. 2020. A large epeiric methanogenic Bambuí sea in the core of Gondwana supercontinent? Geoscience Frontiers, 12(1): 203-218. https://doi.org/10.1016/j.gsf.2020.04.005

CALEGARI, M.R.; MADELLA, M.; VIDAL TORRADO, P.; PESSENDA, L.C.R.; MARQUES, F.A. 2013. Combining phytoliths and soil organic matter in Holocene palaeoenvironmental studies of tropical soils: the example of an oxisol in Brazil. Quaternary International, 287: 47-55. https://doi.org/10.1016/j.quaint.2011.11.012

CALEGARI, M.R.; MADELLA, M.; BRUSTOLIN, L.T.; PESSENDA, L.C.R.; BUSO JR. A.A.; FRANCISQUINI, M.I.; BENASSOLLI, J.A.; VIDAL-TORRADO, P. 2017. Potential of soil phytoliths, organic matter and carbono isotopes for smallscale differentiation of tropical rainforest vegetation: A pilot study from the campos nativos of the Atlantic Forest in Espírito Santo State (Brazil). Quaternary International, 437: 156-164. https://doi.org/10.1016/j. quaint.2016.01.023
CAMARGO, P.B.; TRUMBORE, S.E.; MARTINELLI, L.A.; DAVIDSON, E.A; NEPSTAD, D.C.; VICTORIA, R.L. 1999. Soil carbon dynamics in regrowing forest of eastern Amazonia. Global Change Biology, 5(6): 693-702. https://doi.org/10.1046/ j.1365-2486.1999.00259.x

CAMPOS, D.V.B. 2003. Uso da técnica de ${ }^{13} \mathrm{C} e$ fracionamento fisico da matéria orgânica em solos sob cobertura de pastagens e cana de açúcar na região da Mata Atlântica. Instituto de Agronomia, Universidade Federal Rural do Rio de Janeiro, Seropédica, Tese de Doutorado, $220 \mathrm{p}$.

CAMPOS, M.C.; CHIESSI, C.M.; VOIGT, I.; PIOLA, A.R.; KUHNERT, H.; MULITZA, S. 2017. $\delta 13 \mathrm{C}$ decreases in the upper western South Atlantic during Heinrich Stadials 3 and 2. Climate of the Past, 13(4): 345-358. https://doi.org/10.5194/cp-13-345-2017

CAMPOS, M.C.; CHIESSI, C.M.; VENANCIO, I.M.; PINHO, T.M.; CRIVELLARI, S.; KUHNERT, H.; SCHMIEDL, G.; DÍAZ,R.A.; ALBUQUERQUE, A.L.S.; PORTILHO-RAMOS, R.C.; BAHR, A.; MULITZA, S. 2020. Constraining millennialscale changes in Northern Component Water ventilation in the western tropical South Atlantic. Paleoceanography and Paleoclimatology, 35(7): e2020PA003876. https://doi.org/10.1029/2020PA003876

CERLING, T. E. 1984. The stable isotopic composition of modern soil carbonate and its relationship to climate. Earth and Planetary Science Letters, 71(2): 229-240. https://doi. org/10.1016/0012-821X(84)90089-X

CERLING, T.E.; HARRIS; J.M.; PASSEY, B. H. 2003. Diets of East African bovadae based on stable isotope analysis. Journal of Mammalogy, 84(2): 456-470. https://doi. org/10.1644/1545-1542(2003)084<0456:DO $\mathrm{EABB}>2.0 . \mathrm{CO} ; 2$

CERLING, T.E.; WYNN, J.G.; ANDANJE, S.A.; BIRD, M.I.; KORIR, D.K.; LEVIN, N.E.; MACE, W.; MACHARIA, A.N.; QUADE, J.; REMIEN, C. H. 2011. Woody cover and hominin environments in the past 6 million years. Nature, 476: 51-56. https://doi. org/:10.1016/j.marmicro.2007.02.002 
CHIESSI, C.M.; ULRICH, S.; MULITZA, S.; PÄTZOLD, J.; WEFER, G. 2007. Signature of the Brazil-Malvinas Confluence (Argentine Basin) in the isotopic composition of planktonic foraminifera from surface sediments. Marine Micropaleontology, 64(1-2): 52-66. https://doi.org/10.1016/j. marmicro.2007.02.002

CROWLEY, B.E. 2012. Stable isotope techniques and applications for primatologists. International Journal of Primatology, 33: 673-701. https://doi.org/10.1007/s10764012-9582-7

CRUZ, F.W.; BURNS, S.J.; JERCINOVIC, M.; KARMANN, I.; SHARP, W.D.; VUILLE, M., 2006. Evidence of rainfall 425 variations in Southern Brazil from trace element ratios $(\mathrm{Mg} / \mathrm{Ca}$ and $\mathrm{Sr} / \mathrm{Ca})$ in a Late Pleistocene stalagmite. Earth Planet Science Letters, 71: 2250-2263. https://doi.org/10.1016/j. gca.2007.02.005

DAVEY, S.D.; JENKYNS, H.C. 1999. Carbonisotope stratigraphy of shallow water limestones and implications for the timing of Late Cretaceous sea-level rise and anoxic events (Cenomanian-Turonian of the periAdriatic carbonate platform, Croatia). Eclogae Geologicae Helvetiae, 92: 163-170.

DERRY, L.A. 2010. A burial diagenesis origin for the Ediacaran Shuram-Wonoka carbon isotope anomaly. Earth and Planetary Science Letters, 294(1-2): 152-162. https:// doi.org/10.1016/j.epsl.2010.03.022

DESJARDINS, T.; FILHO, A.C.; MARIOTTI, A.; GIRARDIN, C.; CHAUVEL, A. 1996. Changes of the forest-savanna boundary in Brazilian Amazonia during the Holocene revealed by stable isotope ratios of soil organic carbon. Oecologia, 108: 749-756. https://doi.org/10.1007/BF00329051

DREYBRODT, W. 1988. Processes in Karst Systems. Springer Series in Physical Environment. Springer, Heidelberg, 282 p.

DUPLESSY， J.-C.; SHACKLETON， N.J.; MATTHEWS, R.K.; PRELL, W.; RUDDIMAN, W.F.; CARALP, M.; HENDY, C.H. 1984. 13C record of benthic foraminifera in the last interglacial ocean: implications for the carbon cycle and the global deep water circulation. Quaternary Research, 21(2): 225-243. https://doi.org/10.1016/00335894(84)90099-1

EHLERINGER J.R. 1978. Implications of quantum yield differences on the distributions of $\mathrm{C}_{3}$ and $\mathrm{C}_{4}$ grasses. Oecologia, 31: 255-267. https://doi.org/10.1007/BF00346246

EHLERINGER, J.R.; MONSON, R.K. 1993. Evolutionary and ecological aspects of photosynthetic pathway variation. Annual Reviews in Ecological Systems, 24: 411439. https://doi.org/10.1146/annurev. es.24.110193.002211

EHLERINGER, J.R.; CERLING, T.E.; HELLIKER, B.R. 1997. C 4 photosynthesis, atmospheric $\mathrm{CO}_{2}$, and climate. Oecologia, 112: 285-299. https://doi.org/10.1007/ s004420050311

EIDE, M.; OLSEN, A.; NINNEMANN, U.S.; JOHANNESSEN, T. 2017. A global ocean climatology of preindustrial and modern ocean $\delta^{13} \mathrm{C}$. Global Biogeochemical Cycles, 31(3): 515-534. https://doi. org/10.1002/2016GB005473

FANG, C.; MONCRIEFF, J.B. 2001. The dependence of soil $\mathrm{CO}_{2}$ efflux on temperature. Soil Biology and Biochemistry, 33(2): 155-165. https://doi.org/10.1016/S00380717(00)00125-5

FOHLMEISTER, J.; SCHOLZ, D.; KROMER, B.; MANGINI, A. 2011. Modelling carbon isotopes of carbonates in cave drip water. Geochimica et Cosmochimica Acta, 75(18): 5219-5228. https://doi.org/10.1016/j. gca.2011.06.023

FRANÇA, M.C.; COHEN, M.C.L.; PESSENDA, L.C.R.; ROSSETI, D.F.; LORENTE, F.L.; BUSO JR., A.A.; GUIMARÃES, J.T.F.; FRIAES, Y.; MACARIO, K. 2013. Mangrove vegetation changes on Holocene terraces of the Doce River southeastern Brazil. Catena, 110: 59-69. https://doi.org/10.1016/j. catena.2013.06.011

FRANCISQUIN, M.I.; LIMA, C.M.; PESSENDA, L.C.R; ROSSETTI, D.F.; FRANÇA, M.C.; COHEN, M.C.L. 2014. Relation between 
carbon isotopes of plants and soils on Marajó Island, a large tropical island: implications for interpretation of modern and past vegetation dynamics in the Amazon region. Palaeogeography, Palaeoclimatology, Palaeoecology, 415: 91-104. https://doi. org/10.1016/j.palaeo.2014.03.032

FREITAS, H.A.; PESSENDA, L.C.R; ARAVENA, R.; GOUVEIA, S.E.M.; RIBEIRO A.S.; BOULET, R. 2001. Late Quaternary vegetation dynamics in the southern Amazon Basin inferred from carbon isotopes in soil organic matter. Quaternary Research, 55(1): 39-46. https://doi.org/10.1006/ qres.2000.2192

GAldOS, M.V.; CERRI, C.C.; CERRI, C.E.P. 2009. Soil carbon stocks under burned and unburned sugarcane in Brazil. Geoderma, 153(3/4): 347-352. https://doi.org/10.1016/j. geoderma.2009.08.025

GILLSON, L.; WALDRON, S.; WILLIS, K.J. 2004. Interpretation of soil $\delta^{13} \mathrm{C}$ as an indicator of vegetation change in African savannas. Journal of Vegetation Science, 15: 339-350. https://doi.org/10.1111/j.1654-1103.2004. tb02270.x

GOMES, T.F.; VAN DE BROEK, M.; GOVERS, G.; SILVA, R.W.C.; MORAES, J.M.; CAMARGO, P.B.; MAZZI, E.A.; MARTINELLI, L.A. 2019. Runoff, soil loss and sources of particulate organic carbon delivered to streams by sugarcane and riparian areas: an isotopic approach. Catena, 181: 104083. https://doi.org/10.1016/j. catena.2019.104083

GOUVEIA, S.E.M.; PESSENDA, L.C.R.; ARAVENA, R.; BOULET, R.; SCHEELYBERT, R.; BENDASSOLI, J.A.; RIBEIRO, A.S.; FREITAS, H.A. 2002. Carbon isotopes in charcoal and soils in studies of paleovegetation and climate changes during the late Pleistocene and the Holocene in the southeast and centerwest regions of Brazil. Global Planetary Change, 33 (12): 95-106. https://doi.org/10.1016/S09218181(02)00064-4

GUILLET, B.; FAIVRE，P.; MARIOTTI，A.; KHOBZI, J. 1988. The ${ }^{14} \mathrm{C}$ dates and ${ }^{13} \mathrm{C} /{ }^{12} \mathrm{C}$ ratios of soil organic matter as a means of studying past vegetation in intertropical regions: Examples from Colombia (South America). Palaeogeography Palaeoclimatology., Palaeoecology, 65(12): 51-58. https://doi.org/10.1016/00310182(88)90111-3

GUPTA, B.K.S. 1999. Modern foraminifera. Springer, $371 \mathrm{p}$.

HÄGGI, C.; SAWAKUCHI, A. O.; CHIESSI, C. M.; MULITZA, S., MOLLENHAUER, G.; SAWAKUCHI, H.O.; BAKER, P.A; ZABEL, M.; SCHEFB, E. 2016. Origin, transport and deposition of leaf-wax biomarkers in the Amazon Basin and the adjacent Atlantic. Geochimica et Cosmochimica Acta, 192: 149-165. https://doi.org/10.1016/j. gca.2016.07.002

HÄGGI, C.; CHIESSI, C.M.; MERKEL, U.; MULITZA, S.; PRANGE, M.; SCHULZ, M.; SCHEFU $\beta$, E. 2017. Response of the Amazon rainforest to late Pleistocene climate variability. Earth and Planetary Science Letters, 479: 50-59. https://doi.org/10.1016/j. epsl.2017.09.013

HALVERSON, G.P.; HOFFMAN, P.F.; SCHRAG, D.P.; MALOOF, A.C.; RICE, A.H.N. 2005. Toward a Neoproterozoic composite carbonisotope record. Geological Society of America Bulletin, 117 (9): 1181-1207. https://doi. org/10.1130/B25630.1

HAYES, J.M.; STRAUSS, H.; KAUFMAN, A.J. 1999. The abundance of ${ }^{13} \mathrm{C}$ in marine organic matter and isotopic fractionation in the global biogeochemical cycle of carbon during the past $800 \mathrm{Ma}$. Chemical Geology; 161(1-3): 103-125. https://doi.org/10.1016/ S0009-2541(99)00083-2

HENDY, C.H. 1971. The isotopic geochemistry of speleothems 1 . The calculation of the effects of the different modes of formation on the isotopic composition of speleothems and their applicability as palaeoclimatic indicators. Geochimica et Cosmochimica Acta, 35(8): 801-824. https://doi.org/10.1016/00167037(71)90127-X

HILLAIRE-MARCEL, C.; RAVELO, A. 2007. The use of Oxygen and Carbon isotopes of Foraminifera in Paleoceanography. 
Developments in Marine Geology. In: C. Hillaire-Marcel; A. Vernal (ed.) Proxies in Late Cenozoic Paleoceanography. Burlington, Elsevier Science, p. 735-764.

HOBSON, K. A. 2011. Isotopic ornithology: a perspective. Journal of Ornithology, 152: 49-66. https://doi.org/10.1007/s10336-0110653-x

HOFFMAN, P.F.; SCHRAG, D.P. 2002. The snowball Earth hypothesis: testing the limits of global change. Terra Nova, 14: 129-155. https://doi.org/10.1046/j.13653121.2002.00408.x

HOWE, J.N.W.; PIOTROWSKI, A.M.; NOBLE, T.L.; MULITZA, S.; CHIESSI, C.M.; BAYON, G. 2016. North Atlantic Deep Water Production during the Last Glacial Maximum. Nature Communications, 7(1): 11765. https:// doi.org/10.1038/ncomms 11765

INSTITUTO BRASILEIRO DE GEOGRAFIA E ESTATÍSTICA - IBGE. Sistema IBGE de recuperação automática - SIDRA. 2017. http://www.ibge.br/sidra/ (último acesso em 30/09/2020).

IPCC. 2001. Climate change: The scientific basis. Cambridge, Cambridge University Press. $881 \mathrm{p}$

IPCC. 2019. IPCC Special Report on the Ocean and Cryosphere in a Changing Climate. In press. https://www.ipcc.ch/srocc/

JAQUETO, P.; TRINDADE, R.I.F.; HARTMANN, G.A.; NOVELLO, V.F.; CRUZ, F.W.; KARMANN, I.; STRAUSS, B.E.; FEINBERG， J.M. 2016. Linking speleothem and soil magnetism in the Pau d'Alho cave (central South America). Journal of Geophysical Research: Solid Earth, 121: 2016JB013541. https://doi. org/10.1002/2016JB013541

JASTROW, J.D.; BOUTTON, T.W.; MILLER, R.M. 1996. Carbon dynamics of aggregateassociated organic matter estimated by carbon-13 natural abundance. Soil Science Society of America Journal, 60: 801-807. https://doi.org/10.2136/ sssaj1996.03615995006000030017x
JENKYNS, H.C. 1985. The early Toarcian and Cenomanian-Turonian anoxic events in Europe: comparisons and contrasts. Geologische Rundschau, 74: 505-518. https://doi.org/10.1007/BF01821208

JIANG, G.Q.; WANG, X.Q.; SHI, X.Y.; ZHANG, S.H.; XIAO, S.H.; DONG, J. 2010. Organic carbon isotope constraints on the dissolved organic carbon (DOC) reservoir at the Cryogenian-Ediacaran transition. Earth and Planetary Science Letters, 299(1-2): 159-168. https://doi.org/10.1016/j.epsl.2010.08.031

KAH, L.C.; BARTLEY, J.K.; TEAL, D.A. 2012. Chemostratigraphy of the Late Mesoproterozoic Atar Group, Taoudeni Basin, Mauritania: Muted isotope variability, facies correlation, and global isotopic trends. Precambrian Research, 200203: 82-103. https://doi.org/10.1016/j. precamres.2012.01.011

KASHIYAMA, Y.; OGAWA, N.O.; KURODA, J.; SHIRO, M.; NOMOTO, S.; TADA, R.; KITAZATO, H.; OHKOUCHI, N. 2008. Diazotrophic cyanobacteria as the major photoautotrophs during mid-Cretaceous oceanic anoxic events: Nitrogen and carbon isotopic evidence from sedimentary porphyrin. Organic Geochemistry, 39(5): 532-549. https://doi.org/10.1016/j. orggeochem.2007.11.010

KEELING, C.D. 1979. The Suess effect: ${ }^{13}$ Carbon${ }^{14}$ Carbon interrelations. Environment International, 2(4-6): 229-300. https://doi. org/10.1016/0160-4120(79)90005-9

KNAUTH, L.P.; KENNEDY, M.J. 2009. The late Precambrian greening of the Earth. Nature, 460: 728-732. https://doi.org/10.1038/ nature 08213

KNOOP, W.; WALKER, B. 1985. Interactions of woody and herbaceous vegetation in a southern African savanna. Journal of Ecololy, 73: $235-253$.

KÖHLER， P.; NEHRBASS-AHLES， C.; SCHMITT, J.; STOCKER, T.F.; FICHER, H. 2017. A 156 kyr smoothed history of the atmospheric greenhouse gases $\mathrm{CO}_{2}, \mathrm{CH}_{4}$, and $\mathrm{N}_{2} \mathrm{O}$ and their radiative forcing. Earth System 
Science Data, 9(1): 363-387. https://doi. org/10.5194/essd-9-363-2017

KORTE, C.; KOZUR, H.W. 2010. Carbon-isotope stratigraphy across the Permian-Triassic boundary: A review. Journal of Asian Earth Sciences, 39(4): 215-235. https://doi. org/10.1016/j.jseaes.2010.01.005

KROOPNICK, P. 1985. The distribution of ${ }^{13} \mathrm{C}$ of $\Sigma \mathrm{CO}_{2}$ in the world oceans. Deep Sea Research Part A. Oceanographic Research Papers, 32(1): 57-84. https://doi.org/10.1016/01980149(85)90017-2

KUMP, L.; ARTHUR, A. 1999. Interpreting carbon-isotope excursions: carbonates and organic matter. Chemical Geology, 161: 181-198. https://doi.org/10.1016/S00092541(99)00086-8

LAL, R. 2004. Soil carbon sequestration impacts on global climate change and food security. Science, 304(5677): 1623-1627. https://doi. org/10.1126/science. 1097396

LAPOLA, D.M.; MARTINELLI, L.A.; PERES, C.A.; OMETTO, J.P.H.B.; FERREIRA, M.E.; NOBRE, C.A.; AGUIAR, A.P.D; BUSTAMANTE, M.M.C.; CARDOSO, M.F.; COSTA, M.H.; JOLY, C.A.; LEITE, C.C.; MOUTINHO, P.; SAMPAIO, G.; STRASSBURG, B.B.N.; VIEIRA, I.C.G. 2013. Pervasive transition of the Brazilian land-use system. Nature Climate Change, 4: 27-35. https://doi.org/10.1038/nclimate2056

LUND, D.; TESSIN, A.; HOFFMAN, J.; SCHMITTNER, A. 2015. Southwest Atlantic water mass evolution during the last deglaciation. Paleoceanography, 30(5): 477 494. https://doi.org/10.1002/2014PA002657

LYNCH-STIEGLITZ， J.; STOCKER， T.F.; BROECKER, W.S.; FAIRBANKS, R.G. 1995. The influence of air-sea exchange on the isotopic composition of oceanic carbon: Observations and modeling. Global Biogeochemical Cycles, 9(4): 653-665. https://doi.org/10.1029/95GB02574

MACKENSEN, A.; SCHMIEDL, G. 2019. Stable carbon isotopes in paleoceanography: Atmosphere, oceans, and sediments. Earth-
Science Reviews, 197: 102893. https://doi. org/10.1016/j.earscirev.2019.102893

MACKENSEN, A.; HUBBERTEN, H. W.; BICKERT, T.; FISCHER, G. 1993. The $\delta^{13} \mathrm{C}$ in benthic foraminiferal tests of Fontbotia wuellerstorfi (Schwager) relative to the $\delta^{13} \mathrm{C}$ of dissolved inorganic carbon in southern ocean deep water: implications for glacial ocean circulation models. Paleoceanography, 8(5): 587-610. https://doi.org/10.1029/93PA01291

MAGNUSSON, W.E.; DE ARAÚJO, M.C.; CINTRA, R.; LIMA, A.P.; MARTINELLI, L. A.; SANAIOTTI, T.M.; VASCONCELOS, H.L.; VICTORIA, R.L. 1999. Contributions of $\mathrm{C}_{3}$ and $\mathrm{C}_{4}$ plants to higher trophic levels in the Amazonian savanna. Oecologia, 119: 91-90. https://doi.org/ 10.1007/PL00008821

MAPA. 2012. Plano setorial de mitigação e de adaptação às mudanças climáticas para a consolidação de uma economia de baixa emissão de carbono na agricultura: plano ABC (Agricultura de Baixa Emissão de Carbono). Ministério da Agricultura, Pecuária e Abastecimento, Ministério do Desenvolvimento Agrário, coordenação da Casa Civil da Presidência da República. Brasília, MAPA/ ACS, $173 \mathrm{p}$.

MARTIN, A.; MARRIOTTI, A.; BALESDENT, J.; LAVELlE, P.; VUATTOUX, R. 1990. Estimate of organic matter turnover rate in a savanna soil by ${ }^{13} \mathrm{C}$ natural abundance measurements. Soil Biology and Biochemistry, 22(4): 517-523. https://doi. org/10.1016/0038-0717(90)90188-6

MARTINELLI, I.A.; PESSENDA， L.C.R.; ESPINOSA, E.; CAMARGO, P.B.; TELLES, F.C.; CERRI, C.C.; VICTORIA, R.L.; ARAVENA, R.; RICHEY, J.; TRUMBORE. 1996. Carbon-13 variation with depth in soils of Brazil and climate change during the Quaternary. Oecologia, 106: 376-381. https://doi.org/10.1007/BF00334565

MARTINELLI, L.A.; CAMARGO, P.B.; LARA, L.B.L.S.; VICTORIA, R.L.; ARTAXO, P. 2002. Stable carbon and nitrogen isotopic composition of bulk aerosol particles in $\mathrm{a}_{4}$ plant landscape of southeast Brazil. Atmospheric Environment, 36: 
2427-2432. https://doi.org/10.1016/S13522310(01)00454-X

MARTINELLI, L.A.; OMETTO, J.P.H.B.; FERRAZ, E.S.; VICTORIA, R.L.; CAMARGO, P.B.; MOREIRA, M.Z. 2009. Desvendando questões ambientais com isótopos estáveis. Oficina de Textos, São Paulo, $144 \mathrm{p}$.

MARTINELLI, L.A.; JOLY, C.A.; NOBRE, C.A.; SPAROVEK, G. 2010. A falsa dicotomia entre a preservação da vegetação natural e a produção agropecuária. Biota Neotropica, 10(4). https://doi.org/10.1590/ S1676-06032010000400036

MCDERMOTT, F. 2004. Palaeo-climate reconstruction from stable isotope variations in speleothems: a review. Quaternary Science Reviews, 23(7-8): 901-918. https:// doi.org/10.1016/j.quascirev.2003.06.021

MICKLER, P.J.; STERN, L.A.; BANNER, J.L. 2006. Large Kinect isotope effects in modern speleothems. Geology Society of America Bulletin, 118(1-2): 65-81. https://doi. org/10.1130/B25698.1

MICKLER, P.J.; CARLSON, P.; BANNER, J.L.; BREECKER, D.O.; STERN, L.; GUILFOYLE, A. 2019. Quantifying carbon isotope disequilibrium during in-cave evolution of drip water along discreet flow paths. Geochimica et Cosmochimica Acta, 244: 182-196. https://doi.org/10.1016/j. gca.2018.09.027

MULITZA, S.; RÜHLEMANN, C.; BICKERT, T.; HALE, W.; PÄTZOLD, J.; WEFER, G. 1998. Late Quaternary $\delta^{13} \mathrm{C}$ gradients and carbonate accumulation in the western equatorial Atlantic. Earth and Planetary Science Letters, 155(3-4): 237-249. https:// doi.org/10.1016/S0012-821X(98)00012-0

MULITZA, S.; ARZ, H.; KEMLE-VON MÜCKE, S.; MOOS, C.; NIEBLER, H.-S.; PÄTZOLD, J.; SEGL, M. 1999. The South Atlantic carbon isotope record of planktic foraminifera. In: G. Fisher; G. Wefer (Eds.) Use of Proxies in Paleoceanography. Verlag Berlin Heidelberg, Springer, p. 427-445. https://doi.org/10.1007/978-3-642-586460_1
MULITZA, S.; CHIESSI, C. M.; SCHEFUß, E.; LIPPOLD, J.; WICHMANN, D.; ANTZ, B.; MACKENSEN, A.; PAUL, A.; PRAGE, M.; REHFELD, K.; WERNER, M.; BICKERT, T.; FRANK, N.; KUHNERT, H.; LYNCHSTIEGLITZ, J.; PORTILHO-RAMOS, R.C.; SAWAKUCHI, A.O.; SCHULZ, M.; SCHWENK, T.; TIEDMANN, R.; VANLENKAMP, M.; ZHANG, Y. 2017. Synchronous and proportional deglacial changes in Atlantic meridional overturning and northeast Brazilian precipitation. Paleoceanography, 32(6): 622-633. https:// doi.org/10.1002/2017PA003084

NARDOTO, G.B.; MURRIETA, R.S.; PRATES, L.E.; ADAMS, C.; GARAVELLO, M.E.P.E.; SCHOR, T.; DE MORAES, A.; RINALDI, F.D.; GRAGNANI, J.G.; MOURA, E.A.F.; PAULO, J.D.-N.; MARTINELLI, L.A. 2011. Frozen chicken for wild fish: Nutritional transition in the Brazilian Amazon region determined by carbon and nitrogen stable isotope ratios in fingernails. American Journal of Human Biology, 23: 642-650. https://doi.org/10.1002/ajhb.21192

NATELHOFFER, K.F.; FRY, B. 1988. Controls on natural nitrogen-15 and carbon-13 abundance in forest soil organic matter. Soil Science Society American Journal, 52: 1633-40. https://doi.org/10.2136/ sssaj1988.03615995005200060024x

NOVELLO, V.F.; CRUZ, F.W.; MCGLUE, M.M.; WONG, C.I.; WARD, B.M.; VUILLE, M.; SANTOS, R.A.; JAQUETO, P.; PESSENDA, L.C.R.; ATORRE, T.; RIBEIRO, L.M.A.L.; KARMANN, I.; BARRETO, E. S.; CHENG, H.; EDWARDS, R. L.; PAULA, M.S.; SCHOLZ, D. 2019. Vegetation and environmental changes in tropical South America from the last glacial to the Holocene documented by multiple cave sediment proxies. Earth Planetary Science Letters, 524: 115717. https://doi.org/10.1016/j. eps1.2019.115717

NOVELLO, V.F.; CRUZ, F.W.; VUILLE, M.; CAMPOS, J.L.P.S.; STRÍKIS, N.M.; APÁESTEGUI, J.; MOQUET, J.S.; AZEVEDO, V.; AMPUERO, A.; UTIDA, G.; WANG, X.; PAULA-SANTOS, G.M.; JAQUETO, P.; PESSENDA, L.C.R.; 
BREECKER, D. O.; KARMANN, I. 2021. Investigating $\delta^{13} \mathrm{C}$ values in stalagmites from tropical South America for the last two millennia, Quaternary Science Reviews, 255: 106822. https://doi.org/10.1016/j. quascirev.2021.106822

OLIVEIRA， D.M.S.; CHRISTIAN, K.P.; DAVIESD, A.; CHERUBIN, M.R.; FRANCO, A.L.C.; CERRI, C.L. 2016. Soil carbon changes in areas undergoing expansion of sugarcane into pastures in south-central Brazil. Agriculture, Ecosystems and Environment, 228: 38-48. https://doi. org/10.1016/j.agee.2016.05.005

OPPO, D. W.; CURRY, W.B.; MCMANUS, J.F. 2015. What do benthic $\delta^{13} \mathrm{C}$ and $\delta^{18} \mathrm{O}$ data tell us about Atlantic circulation during Heinrich Stadial 1?. Paleoceanography, 30(4): 353368. https://doi.org/10.1002/2014PA002667

PAULA-SANTOS, G.M.; CAMPANHA, G.A.C.; FALEIROS, F.M.; HOLLANDA, M.H.M.B; RODRIGUES, S.W.O. 2021. Carbon isotope variations of high magnitude recorded in carbonate rocks from the Stenian-Tonian Lajeado Group, Southeast Brazil. Journal of South America Earth Sciences, 109, 103268. https://doi.org/10.1016/j.jsames.2021.103268

PESSENDA，L.C.R.; ARAVENA，R.; MELFI, A.J.; TELLES, E.C.C.; BOULET, R.; VALENCIA, E.P.E.; TOMAZELLO, M. 1996a. The use of carbon isotopes $\left(C^{-13}, C^{-14}\right)$ in soil to evaluate vegetation changes during the Holocene in Central Brazil. Radiocarbon, 38 (2): 191-201. https://doi.org/10.1017/ S0033822200017562

PESSENDA, L.C.R.; VALENCIA, E.P.E.; CAMARGO, P.B.; TELLES, E.C.C.; MARTINELLI, L.A.; CERRI, C.C.; ARAVENA, R.; ROZANSKI, K. 1996 b. Natural radiocarbon measurements in Brazilian soils developed on basic rocks. Radiocarbon, 38 (2): 203-208. https://doi. org/10.1017/S0033822200017574

PESSENDA, L.C.R; GOMES, B.M.; ARAVENA, R.; RIBEIRO, A.S.; BOULET, R.; GOUVEIA, S.E.M. 1998. The carbon isotope record in soils along a forestcerrado ecosystem transect: implications for vegetation changes in the Rondônia State, southwestern Brazilian Amazon region. The Holocene, 8(5): 599-603. https://doi. org/10.1191/095968398673187182

PESSENDA, L.C.R.; BOULET, R.; ARAVENA, R.; ROSOLEN, V.; GOUVEIA, S.E.M.; RIBEIRO, A.S.; LAMOTTE, M. 2001a. Origin and dynamics of soil organic matter and vegetation changes during the Holocene in a forest-savanna transition zone, Brazilian Amazon Region. The Holocene, 11(2): 250-4. https://doi. org/10.1191/095968301668898509

PESSENDA, L.C.R.; GOUVEIA, S.E.M.; ARAVENA, R. 2001b. Radiocarbon dating of total soil organic matter and humin fraction and its comparison with ${ }^{14} \mathrm{C}$ ages of fossil charcoal. Radiocarbon, 43 (2B): 595-601. https://doi.org/10.1017/S0033822200041242

PESSENDA, L.C.R; RIBEIRO, A.S.; GOUVEIA, S.E.; ARAVENA, R; BOULET, R.; BENDASSOLI, J.A. 2004. Vegetation dynamics during the late Pleistocene in the Barreirinhas region, Maranhão State, Northeastern Brazil, based on carbon isotopes in soil organic matter. Quaternary Research, 62(2): 183-193. https://doi.org/10.1016/j. yqres.2004.06.003

PESSENDA, L.C.R.; LEDRU, M.P.; GOUVEIA, S.E.M; ARAVENA, R.; RIBEIRO, A.S.; BENDASSOLLI, J.A.; BOULET, R. 2005. Holocene palaeoenvironmental reconstruction in northeastern Brazil inferred from pollen, charcoal and carbon isotope records. The Holocene, 15(6): 814-22. https:// doi.org/10.1191/0959683605h1855ra

PESSENDA， L.C.R.; DE OLIVEIRA, P.E.; MOFATTO, M.; MEDEIROS, V.B.M.; GARCIA, R.J.F.; ARAVENA, R.; BENDASSOLLI, J.A.; LEITE, A.Z.; SAAD, A.R.; ETCHEBEHERE, M.L. 2009. The evolution of a tropical rainforest/grassland mosaic in southeastern Brazil since 28,000 ${ }^{14} \mathrm{C}$ yr BP based on carbon isotopes and pollen records. Quaternary Research, 71(3): 437-459. https://doi.org/10.1016/j. yqres.2009.01.008

PESSENDA, L.C.R.; SAIA, S.E.M.G.; GOUVEIA, S.E.M.; LEDRU, M.-P.; SIFFEDINE, A.; AMARAL, P.G.C.; BENDASSOLLI, J.A. 
2010. Last millennium environmental changes and climate inferences in the Southeastern Atlantic Forest, Brazil. Anais da Academia Brasileira de Ciências, 82 (3): 717-729. https://doi.org/10.1590/S000137652010000300019

PESSENDA, L.C.R.; VIDOTTO, E.; OLIVEIRA, P.E.O.; BUSO JR., A.A.; COHEN, M.C.L.; ROSETTI, D.F.; RICARDIBRANCO, F.; BENDASSOLLI, J.A. 2012. Late Quaternaary vegetation and coastal environmental changes at Ilha do Cardoso mangrove, southeastern Brazil. Pelaeogeography, Palaeoclimatology, Palaeoecology, 363-364: 57-68. https://doi. org/10.1016/j.palaeo.2012.08.014

POLAG, D.; SCHOLZ, D.; MÜHLINGHAUS, C.; SPÖTL, C.; SCHRÖDER-RITZRAU, A.; SEGL, M.; MANGINI, A. 2010. Stable isotope fractionation in speleothems: Laboratory experiments. Chemical Geology, 279(1-2): 31-39. https://doi.org/10.1016/j. chemgeo.2010.09.016

POLLEY, H.W.; JOHNSONH, B.; MAYEUXH, S. 1992. Carbon dioxide and water fluxes of $\mathrm{C}_{3}$ annuals and $\mathrm{C}_{3}$ and $\mathrm{C}_{4}$ perennials at subambient $\mathrm{CO}_{2}$ concentrations. Functional Ecology, 6: 693-703. https://doi. org/10.2307/2389966

POLLEY, H.W.; JOHNSONH, B.; MARINOB, D; MAYEUXH, S. 1993. Increase in $\mathrm{C}_{3}$ plant water-use efficiency and biomass over Glacial to present $\mathrm{CO}_{2}$ concentrations. Nature, 361: 61-64. https://doi.org/10.1038/361061a0

PORTILHO-RAMOS, R.C.; CRUZ, A.; BARBOSA, C.; RATHBURN, A.E.; MULITZA, S.; VENANCIO, I.M.; SCHWENK, T.; RÜHLEMANN, C.; VIDAL, L.; CHIESSI, C.M.; SILVEIRA, C.S. 2018. Methane release from the southern Brazilian margin during the last glacial. Scientific reports, 8(1): 1-9. https://doi.org/10.1038/ s41598-018-24420-0

POWELL, R. L.; YOO, E-Y.; STILL, C. J. 2012. Vegetation and soil carbon-13 isoscapes for South America: integrating remote sensing and ecosystem isotope measurements. Ecosphere, 3(11): 109. https://doi. org/10.1890/ES12-00162.1
QUADE, J.; CERLING, T.E.; BOWMAN, J.R. 1989. Systematic variations in the carbon and oxygen isotopic composition of pedogenic carbonate along transects in the Southern Great Basin, United States. Geological Society of America Bulletin, 101(4): 464-475. https://doi.org/10.1130/00167606(1989)101<0464:SVITCA>2.3.CO;2

RAVELO, A.; FAIRBANKS, R. 1995. Carbon isotopic fractionation in multiple species of planktonic foraminifera from coretops in the tropical Atlantic. The Journal of Foraminiferal Research, 25(1): 53-74. https://doi.org/10.2113/gsjfr.25.1.53

RAVEN, J.; CALDEIRA, K.; ELDERFIELD, H.; HOEGH-GULDBERG, O.; LISS, P.; RIEBESESELL, U.; SHEPHERD, J.; TURLEY, C.; WATSON, A. 2005. Ocean acidification due to increasing atmospheric carbon dioxide. The Royal Society, 2005. 0854036172. ISBN 0854036172

ROHLING, E. J.; COOKE, S. 1999. Stable oxygen and carbon isotopes in foraminiferal carbonate shells. In: B.K. Sen Gupta (Ed.) Modern foraminifera. Dordrecht, Springer, 239-258 p. https://doi.org/10.1007/0-30648104-9_14

RHOADES, C.C.; ECKERT, G. E.; COLEMAN, D.C. 2000. Soil carbon differences among forest, agriculture, and secondary vegetation in lower montane Ecuador. Ecological Applications 10: 497-505. https://doi. org/10.2307/2641109

ROMANEK, C. S.; GROSSMAN, E.L.; MORSE, J. W. 1992. Carbon isotopic fractionation in synthetic aragonite and calcite: effects of temperature and precipitation rate. Geochimica et Cosmochimica Acta, 56(1): 419-430. https://doi.org/10.1016/00167037(92)90142-6

ROMANOU, A.; MARSHALL, J.; KELLEY, M.; SCOTT, J. 2017. Role of the ocean's AMOC in setting the uptake efficiency of transient tracers. Geophysical Research Letters, 44(11): 5590-5598. https://doi. org/10.1002/2017GL072972

ROSSETI, D.; COHEN, M.C.L.; PESSENDA, L.C.R. 2017. Vegetation change in 
Southwestern Amazonia (Brazil) and relationship to the late Pleistocene and Holocene climate. Radiocarbon, 59(1): 6989. https://doi.org/10.1017/RDC.2016.107

SAIA, S.; PESSENDA, L.; GOUVEIA, S.; ARAVENA, R.; BENDASSOLLI, J.; PESSENDA, L.C.R. 2008. Last glacial maximum (LGM) vegetation changes in the Atlantic Forest, southeastern Brazil. Quaternary International, 184(1): 195-201. https://doi.org/10.1016/j.quaint.2007.06.029

SANSJOFRE, P.; ADER, M.; TRINDADE, R.I.F.; ELIE, M.; LYONS, J.; CARTIGNY, P.; NOGUEIRA, A.C.R. 2011. A carbon isotope challenge to the snowball Earth. Nature, 478: 93-96. https://doi.org/10.1038/nature10499

SANTOS, T.P.; LESSA, D.O.; VENANCIO, I.M.; CHIESSI, C.M.; MULITZA, S.; KUHNERT, H.; ALBUQUERQUE, A.L.S. 2017a. The impact of the AMOC resumption in the western South Atlantic thermocline at the onset of the Last Interglacial. Geophysical Research Letters, 44(22): 11547-11554. https://doi.org/10.1002/2017GL074457

SANTOS, T.P.; LESSA, D.O.; VENANCIO, I.M.; CHIESSI, C.M.; MULITZA, S.; KUHNERT, GOVIN, A.; MACHADO, T.; COSTA, K.B.; TOLEDO, F.; DIAS, B.B.; ALBUQUERQUE, A.L.S. 2017b. Prolonged warming of the Brazil Current precedes deglaciations. Earth and Planetary Science Letters, 463: 1-12. https://doi.org/10.1016/j. epsl.2017.01.014

SARMIENTO, J.; TOGGWEILER, J.; NAJJAR, R. 1988. Ocean carbon-cycle dynamics and atmospheric $p \mathrm{CO}^{2}$. Philosophical Transactions of the Royal Society of London. Series A, Mathematical and Physical Sciences, 325 (1583): 3-21. https://doi. org/10.1098/rsta.1988.0039

SHACKLETON, N. $1977 .{ }^{13} \mathrm{C}$ in Uvigerina: Tropical rain forest history and the equatorial Pacific carbonate dissolution cycle. In: N.R. Anderser; A. Malahoff (ed.) The Fate of Fossil Fuel in the Oceans. New York, Plenum, p. 401-427. https://doi.org/10.1594/ PANGAEA.692091
SCHEEL-YBERT, R.; GOUVEIA, S.E.M.; PESSENDA， L.C.R.; ARAVENA， R.; COUTINHO, L.M.; BOULET, R. 2003. Holocene palaeoenvironmental Evolution in the São Paulo State (Brazil), based on anthracology and soil $\mathrm{d} 13 \mathrm{C}$ analysis. The Holocene, 13(1): 73-81. https://doi. org/10.1191/0959683603hl596rp

SMITH, B.N.; EPSTEIN, S. 1971. Two categories of ${ }^{13} \mathrm{C} /{ }^{12} \mathrm{C}$ rations for higher plants. Plant Physiology, 47(3): 380-384. https://doi. org/10.1104/pp.47.3.380

SPERO, H.J. 1992. Do planktic foraminifera accurately record shifts in the carbon isotopic composition of seawater $\mathrm{\Sigma CO}_{2}$ ? Marine Micropaleontology, 19(4): 275-285. https:// doi.org/10.1016/0377-8398(92)90033-G

SPERO, H.J.; LEA, D.W. 1993. Intraspecific stable isotope variability in the planktic foraminifera Globigerinoides sacculifer: Results from laboratory experiments. Marine Micropaleontology, 22(3): 221-234. https:// doi.org/10.1016/0377-8398(93)90045-Y

SPERO, H.J.; BIJMA, J.; LEA, D.W.; BEMIS, B.E. 1997. Effect of seawater carbonate concentration on foraminiferal carbon and oxygen isotopes. Nature, 390(6659), 497500. https://doi.org/10.1038/37333

STEPH, S.; REGENBERG, M.; TIEDEMANN, R.; MULITZA, S.; NÜRNBERG, D. 2009. Stable isotopes of planktonic foraminifera from tropical Atlantic/Caribbean core-tops: Implications for reconstructing upper ocean stratification. Marine Micropaleontology, 71(1-2): 1-19. https://doi.org/10.1016/j. marmicro.2008.12.004

SUITS, N.S.; DENNING, A. S.; BERRY, J.A.; STILL, C. J.; KADUK, J.; MILLER, J. B.; BAKER, I. T. 2005. Simulation of carbon isotope discrimination of the terrestrial biosphere. Global Biogeochemical Cycles, 19. https://doi.org/10.1029/2003GB002141

SUN, S.; SCHEFUß, E.; MULITZA, S.; CHIESSI, C.M.; SAWAKUCHI, A.O.; ZABEL, M.; BAKER, P.A.; HEFTER, J.; MOLLENHAUER, G. 2017. Origin and processing of terrestrial organic carbon in the Amazon system: lignin phenols in river, 
shelf, and fan sediments. Biogeosciences, 14(9): 2495-2517. https://doi.org/10.5194/ bg-14-2495-2017

TARRÉ, R.M.; MACEDO, R.; CANTARUTTI, R.B.; RESENDE, C.P.; PEREIRA, J. M.; FERREIRA, E.; ALVES, B.J.R.; URQUIAGA, S.; BODDEY, R.M. 2001. The effect of the presence of a forage legume on nitrogen and carbon levels in soils under Brachiaria pastures in the Atlantic forest region of the south of Bahia, Brazil. Plant and Soil, 234: 15-26. https://doi. org/10.1023/A:1010533721740

THEODOR, M.; SCHMIEDL, G.; JORISSEN, F.; MACKENSEN, A. 2016. Stable carbon isotope gradients in benthic foraminifera as proxy for organic carbon fluxes in the Mediterranean Sea. Biogeosciences, 13(23): 6385-6404. https://doi.org/10.5194/bg-136385-2016

TRUMBORE, S.E.; DAVIDSON, E.A.; CAMARGO, P.B.; NEPSTAD, D.C.; MARTINELLI, L.A. 1995. Belowground cycling of carbon in forests and pastures of eastern Amazonia. Global Biogeochemical Cycles, 9(4): 515-528. https://doi. org/10.1029/95GB02148

VENANCIO, I.M.; BELEM, A.L.; SANTOS, T.P.; LESSA, D.O.; ALBUQUERQUE, A.L.S.; MULITZA, S.; SCHULZ, M.; KUCERA, M. 2017. Calcification depths of planktonic foraminifera from the southwestern Atlantic derived from oxygen isotope analyses of sediment trap material. Marine Micropaleontology, 136: 37-50. https://doi. org/10.1016/j.marmicro.2017.08.006

VICTORIA, R.L.; FERNANDES, F.; MARTINELLI, L.A.; PICOLLO, M.C.; DE CARMARGO, P.B.; TRUMBORE, S.E. 1995. Past vegetation changes in the Brazilian Pantanal arboreal-grassy savanna ecotone by using carbon isotopes in the soil organic matter. Global Change Biology, 1: 165-171. https://doi.org/10.1111/j.1365-2486.1995. tb00018.x

VIDOTTO, E.; PESSENDA, L.C.R.; RIBEIRO, A.S.; FREITAS, H.A.; BENTASSOLI, J.A. 2007. Dinâmica do ecótono florestacampo no sul do estado do Amazonas no
Holoceno, através de estudos isotópicos e fitossociológicos. Acta Amazonica, 37(3): 385-400. https://doi.org/10.1590/S004459672007000300010

VITORELLO V.A.; CERRI, C.C.; ANDREUX F.; FELLER C.; VICTÓRIA, R.L. 1989. Organic Matter and Natural Carbon-13 Distribution in Forested and Cultivated Oxisols. Soil Science Society of America Journal, 53(3): 773-778. https://doi. org/10.1002/2017PA003095

VOIGT, I.; CRUZ, A.; MULITZA, S.; CHIESSI, C.; MACKENSEN, A.; LIPPOLD, J.; ANTZ, B.; ZABEL, M.; ZHANG, Y.; BARBOSA, C.F.; TISSERAND, A.A. 2017. Variability in mid-depth ventilation of the western Atlantic Ocean during the last deglaciation. Paleoceanography, 32(9): 948-965. https:// doi.org/10.5194/cp-8-1309-2012

VUILLE，M.; BURNS， S.J.; TAYLOR，B.L.; CRUZ, F.W.; BIRD, B.W.; ABBOTT, M. B.; KANNER, L.C.; CHENG, H.; NOVELLO, V.F. 2012. A review of the South American Monsoon history as recorded in stable isotopic proxies over the past two millennia. Climate Past, 8(4): 1309-1321. https://doi. org/10.5194/cp-8-1309-2012

WANTZEN, K.M.; MACHADO, F.A.; VOSS, M.; BORISS, H.; JUNK, J. 2002. Seasonal isotopic shifts in fish of the Pantanal wetland Brazil. Aquatic Sciences, 64: 239-251. https://doi.org/10.1007/PL00013196

WARD, B.W.; WONG, C.I.; NOVELLO, V.F.; MCGEE, D.; SANTOS, R.V.; SILVA, L.C.R.; CRUZ, F.W.; WANG, X.; EDWARDS, R.L.; CHENG, H. 2019. Monsoon System and local moisture variability from speleothem $\delta^{18} \mathrm{O}$ and ${ }^{87} \mathrm{Sr} /{ }^{86} \mathrm{Sr}$ records, Quaternary Science Reviews, 210: 51-63. https://doi. org/10.1016/j.quascirev.2019.02.019

WEDIN，D.A.; TIESZEN，L.L.; DEWEY，B.; PASTOR, J. 1995. Carbon isotope dynamics during grass decomposition and soil organic matter formation. Ecology 76: 1383-1392. https://doi.org/10.2307/1938142

WEFER, G.; BERGER, W. H. 1991. Isotope paleontology: growth and composition of extant calcareous species. Marine 
geology, 100(1-4): 207-248. https://doi. org/10.1016/0025-3227(91)90234-U

WORTHAM, B.E.; WONG, C.I.; SILVA, L.C.R.; MCGEE, D.; MONTAÑEZ, I.P.; RASBURY, E.T.; COOPER, K.M.; SHARP, W.D.; GLESSNER, J.J.G.; SANTOS, R. V. 2017. Assessing response of local moisture conditions in central Brazil to variability in regional monsoon intensity using speleothem ${ }^{87} \mathrm{Sr} /{ }^{86} \mathrm{Sr}$ values. Earth and Planetary Science Letters, 463: 310-322. https://doi. org/10.1016/j.eps1.2017.01.034

ZHU, X.; DI, D.; MA, M.; SHI, W. 2019. Stable Isotopes in Greenhouse Gases from Soil: A Review of Theory and Application. MDPI Atmosphere, 10(7): 377-390. https://doi. org/10.3390/atmos10070377

\section{Endereço dos autores:}

Valdir F. Novello, Giselle Utida e Francisco William da Cruz - Instituto de Geociências, Universidade de São Paulo, Rua do Lago, 562, CEP 05508-08, São Paulo, Brasil. E-mails: vfnovello@gmail.com, giselleutida@hotmail.com, cbill@usp.br

Marília de Carvalho Campos - Instituto de Geociências, Universidade de São Paulo, Rua do Lago, 562, CEP 05508-08, São Paulo, Brasil / Escola de Artes, Ciências e Humanidades, Universidade de São Paulo, Rua Arlindo Bettio, 1000, CEP 03828-000, São Paulo, Brasil.E-mail: marilia.carvalho.campos@usp.br

Cristiano Mazur Chiessi - Escola de Artes, Ciências e Humanidades, Universidade de São Paulo, Rua Arlindo Bettio, 1000, CEP 03828-000, São Paulo, Brasil E-mail: chiessi@usp.br

Gustavo Macedo de Paula-Santos-Faculty of Geosciences and MARUM-Center for Marine Environmental Sciences, University of Bremen, Leobener Strasse 8, 28359, Bremen, Alemanha.E-mail: gustavomps@ yahoo.com.br

Luiz Carlos Ruiz Pessenda e Taciana Gomes - Centro de Energia Nuclear na Agricultura, Universidade de São Paulo, Av. Centenário, 303, CEP 13416-000, Piracicaba, Brasil.E-mails: pessenda@cena.usp.br, tacy0906@gmail.com

Artigo submetido em 30 de outubro de 2020, aceito em 6 de janeiro de 2021. 